\title{
HOPF CONSTRUCTIONS AND HIGHER PROJECTIVE PLANES FOR ITERATED LOOP SPACES
}

\author{
NICHOLAS J. KUHN, MICHAEL SLACK, ANL FRANK WILLIAMS
}

\begin{abstract}
We define a category, $\mathscr{H}_{p}{ }^{n}$ (for each $n$ and $p$ ), of spaces with strong homotopy commutativity properties. These spaces have just enough structure to define the $\bmod p$ Dyer-Lashof operations for $n$-fold loop spaces. The category $\mathscr{H}_{p}^{n}$ is very convenient for applications since its objects and morphisms are defined in a homotopy invariant way. We then define a functor, $P_{p}^{n}$, from $\mathscr{H}_{p}^{n}$ to the homotopy category of spaces and show $P_{p}^{n}$ to be left adjoint to the $n$-fold loop space functor. We then show how one can exploit this adjointness in cohomological calculations to yield new results about iterated loop spaces.
\end{abstract}

\section{INTRODUCTION}

The Hopf construction (see [BT, Sf]) has been a fundamental tool in unstable homotopy theory; it has been used extensively in the study of (one-fold) loop spaces and $\mathrm{H}$-spaces. Examples of beautiful applications of the Hopf construction can be found in [Ad, $\mathrm{Hu}, \mathrm{Ka}, \mathrm{Li}, \mathrm{Th} 2$ ], and, of course, in the construction of the Hopf maps $S^{3} \rightarrow S^{2}, S^{7} \rightarrow S^{4}$, and $S^{15} \rightarrow S^{8}$. The main objective of this paper is to extend some of the techniques associated to the Hopf construction to the more general framework of $n$-fold loop spaces $(1 \leq n \leq \infty)$ and $H_{p}^{n}$-spaces (which are the analogue of H-spaces). Thus we forge a link between the Hopf construction point of view in H-space theory and the theory of iterated loop spaces.

The main results of this paper can be summarized as follows. In $\S 2$ we define a category, $\mathscr{H}_{p}{ }^{n}$, for each $2 \leq p \leq \infty$ and $0 \leq n \leq \infty$. The objects of $\mathscr{H}_{p}^{n}$ are called $H_{p}^{n}$-spaces; an $H_{p}^{0}$-space is just an ordinary space, an $H_{2}^{1}$-space is an $\mathrm{H}$-space, and for larger $n$ and $p$, an $H_{p}^{n}$-space is an $\mathrm{H}$-space which possesses some additional homotopy commutativity. More precisely, let $C_{n} X$ be the usual configuration space approximation to $\Omega^{n} \Sigma^{n} X$ with $p$ th filtration $C_{n, p} X$, and let $\widetilde{C}_{n, p} X$ be the cofiber (mapping cone) of the inclusion $X \hookrightarrow C_{n, p} X$. Then an $H_{p}^{n}$-space structure on $X$ is a map $\theta_{n}^{X}: C_{n, p} X \rightarrow X$ extending the identity on $X$. For example, an $n$-fold loop space will be an $H_{p}^{n}$-space for all $p$. A morphism in our category is the homotopy class of a map which preserves $H_{p}^{n}$-structures, together with the homotopy class of a homotopy which makes

Received by the editors May 24, 1993.

1991 Mathematics Subject Classification. Primary 55P45, 55P35; Secondary 55S12, 55P47, 55Q25.

Research of the first author partially supported by the NSF. 
the requisite diagram commute. Thus one should view $\mathscr{H}_{p}{ }^{n}$ as a "homotopy category of $H_{p}^{n}$-spaces" (although it is not defined as the homotopy category of an underlying category when $n \geq 1$ ).

In $\S 3$ we define functors $\Omega^{n}: \mathscr{H}_{p}^{0} \rightarrow \mathscr{H}_{p}^{n}$ and $P_{p}^{n}: \mathscr{H}_{p}^{n} \rightarrow \mathscr{H}_{p}^{0}$ (for $1 \leq n<$ $\infty)$. The functor $\Omega^{n}$ is the usual $n$-fold loop space functor, and $P_{p}^{n}$ is an appropriate higher "projective plane" construction. By definition, $P_{p}^{n} X$ is the cofiber of a natural map

$$
h: \Sigma^{n} \widetilde{C}_{n, p} X \rightarrow \Sigma^{n} X .
$$

In the case that $n=1$ and $p=2$, the homotopy equivalences $X * X \simeq$ $\Sigma(X \wedge X) \simeq \Sigma \widetilde{C}_{1,2} X$ (where $X * X$ is the (reduced) join of $X$ with itself) yield a map

$$
h: X * X \rightarrow \Sigma X
$$

that agrees (up to homotopy) after one suspension with the usual Hopf map [BT, Sf]. Thus there is a homotopy equivalence between $\Sigma P_{2}^{1} X$ and $\Sigma P_{2} X$, where $P_{2} X$ is the projective plane of an H-space $X$ which arises from the Hopf construction.

In $\S 4$ we then prove what is probably the most important result of the paper.

Theorem 4.9. $P_{p}^{n}$ is left adjoint to $\Omega^{n}$.

En route to proving this theorem we establish an important compatibility result between the various versions of $h$ that result from different values of $n$.

Theorem 4.1.

(i) Suppose $k \geq 1$, and let $\left(X, \theta_{k}^{X}\right) \in \mathrm{Ob} \mathscr{H}_{p}^{k}$. Then the constructions for $\left(X, \theta_{k}^{X}\right)$ and $\left(\Omega^{n} X, \theta_{n+k}^{\Omega^{n} X}\right) \in \mathrm{Ob} \mathscr{H}_{p}{ }^{n+k}$ fit into the following naturally (strictly) commutative diagram:

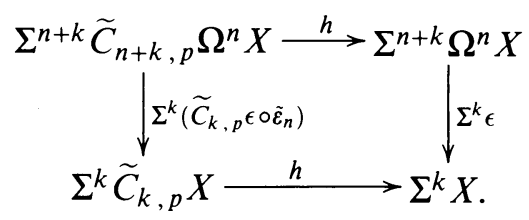

(ii) If $X \in \mathrm{Ob} \mathscr{H}_{p}^{0}$, then for $\left(\Omega X, \theta_{1}^{\Omega X}\right) \in \mathrm{Ob} \mathscr{H}_{p}{ }^{1}$, the following composite is naturally null-homotopic:

$$
\Sigma \widetilde{C}_{1, p} \Omega X \stackrel{h}{\longrightarrow} \Sigma \Omega X \stackrel{\epsilon}{\longrightarrow} X .
$$

Here $\epsilon$ and $\tilde{\varepsilon}_{n}$ are appropriate evaluation maps. This compatibility result is similar to the main result of [Kul].

In $\S 5$ we discuss how the results of $\S 3$ extend to the case $n=\infty$. We have adjoint functors $\mathbf{P}_{\mathbf{p}}: \mathscr{H}_{p}^{\infty} \rightarrow \mathscr{H} \mathscr{S}$ and $\Omega^{\infty}: \mathscr{H} \mathscr{S} \rightarrow \mathscr{H}_{p}^{\infty}$, where $\mathscr{H} \mathscr{S}$ is the homotopy category of spectra, and $\mathbf{P}_{\mathbf{p}} X$ will be the cofiber of a generalized Kahn-Priddy map

$$
h: \Sigma^{\infty} \widetilde{C}_{\infty, p} X \rightarrow \Sigma^{\infty} X
$$

of the sort studied in [Ku2].

In $\S 6$ we turn to the implications of the above on the calculation of the $\bmod 2$ cohomology of $P_{2}^{n} X$. Our motivation comes from the Hopf construction. A 
fundamental result of Thomas [Th1, Sf] is that in the mod 2 cohomology of the cofibration sequence

$$
X * X \stackrel{H}{\longrightarrow} \Sigma X \stackrel{I}{\longrightarrow} P_{2} X \stackrel{J}{\longrightarrow} \Sigma(X * X),
$$

one has $\bar{x}^{2}=J^{*}\left(I^{*}(\bar{x}) \otimes I^{*}(\bar{x})\right)$ for all $\bar{x} \in \widetilde{H}^{*}\left(P_{2} X ; \mathbf{F}_{2}\right)$. A proof of a generalized version of this result can found in Appendix B.

To generalize this, we first note that an $H_{2}^{n}$-space structure on $X$ is just enough so that the mod 2 homology of $X$ admits certain Dyer-Lashof operations [AK, Br, CLM, DL] which are detected by the map $h$. Then Theorems 4.1 and 4.9 are used to prove the following theorem, using the cofibration sequence

$$
\Sigma^{n} \widetilde{C}_{n, 2} X \stackrel{h}{\longrightarrow} \Sigma^{n} X \stackrel{i}{\longrightarrow} P_{2}^{n} X \stackrel{j}{\longrightarrow} \Sigma^{n+1} \widetilde{C}_{n, 2} X,
$$

defined for an $H_{2}^{n}$-space $X$.

Theorem 6.10. Suppose $X \in \mathrm{Ob} \mathscr{H}_{2}^{n}$. If $\bar{x} \in \widetilde{H}^{q+n}\left(P_{2}^{n} X ; \mathbf{F}_{2}\right)$, then

$$
S q^{q+n} \bar{x}=j^{*}\left(\sigma^{n+1}\left(\tilde{Q}_{n-1} x\right)\right),
$$

where $i^{*} \bar{x}=\sigma^{n} x$ defines $x \in \widetilde{H}^{q}\left(X ; \mathbf{F}_{2}\right)$. In particular, if $S q^{q+n} \bar{x}=0$, then there is an element $y \in \widetilde{H}^{2 q+n-1}\left(X ; \mathbf{F}_{2}\right)$ satisfying $\langle x, v\rangle=\left\langle y, Q_{n-1} v\right\rangle$ for all $v \in \widetilde{H}_{q}\left(X ; \mathbf{F}_{2}\right)$.

Here $\widetilde{Q}_{n-1} x$ is a certain well-defined element of $\widetilde{H}^{n-1+2 q}\left(\widetilde{C}_{n, 2} X ; \mathbf{F}_{2}\right)$, and $Q_{n-1}$ is the usual homology Dyer-Lashof operation. There is also an appropriate $n=\infty$ version of this theorem (Corollary 6.11 ), as well as an odd primary version we leave for future writeup.

Roughly put, this theorem says that if many Dyer-Lashof operations are zero in $\widetilde{H}_{*}\left(X ; \mathbf{F}_{2}\right)$, then many Steenrod operations will be nonzero in $\widetilde{H}^{*}\left(P_{2}^{n} X ; \mathbf{F}_{2}\right)$. When $n=\infty$ and $X$ is an infinite loop space, one obtains information about the cohomology of the infinite delooping of $X$ from $\widetilde{H}_{*}\left(X ; \mathbf{F}_{2}\right)$, viewed as an algebra over the Dyer-Lashof algebra. This often yields results more easily than the Miller spectral sequence [Mi], in situations analogous to ones in which projective plane methods are preferable to the use of the Eilenberg-Moore spectral sequence.

In this spirit, the second author has used the results of this paper to prove the following theorem, which appears separately in [S12].

Theorem [S12]. If $X$ is a connected $H_{p}^{\infty}$-space (of finite type), and all of the Dyer-Lashof operations are trivial on the $\bmod p$ homology of $X$, then $X$ is $\bmod p$ homotopy equivalent to a product of Eilenberg-Mac Lane spaces.

Section 7 contains some examples and applications of our constructions. In particular, we give a simple proof that the 3-connected cover of $S^{3}$ is not an $\mathrm{H}_{2}^{4}$-space, illustrating how one can use Theorem 6.10.

In Appendix A we discuss the relationship between our construction and the usual Hopf construction when we restrict to the case $n=1$ and $p=2$. In Appendix B, we give a proof of Thomas' theorem which is valid for any cohomology theory.

The authors would like to thank Yutaka Hemmi, who explained his work in [He] to us. In particular, he has constructed a space which is closely related to 
our $P_{2}^{2} X$ by using the Hopf construction. This was the original impetus for the ideas that led to this paper. We would also like to thank Brayton Gray, Tom Hunter, Jim Lin, Haynes Miller, Nori Minami, David Pengelley, and Bill Richter for contributing in one way or another to our understanding of this work.

We briefly review some of the conventions and notation used in this paper. All spaces are assumed to be based compactly generated weak Hausdorff spaces of finite type, and all maps are based maps. Basepoints (denoted by $*$ ) will be nondegenerate, in the sense that $(X, *)$ is an NDR-pair [St] for a based space $X$. Spectra will be denoted in boldface type.

\section{The CATEgory $\mathscr{H}_{p}^{n}$}

The purpose of this section is to define the categories, $\mathscr{H}_{p}^{n}$, of $H_{p}^{n}$-spaces and $H_{p}^{n}$-maps which are used in this paper. Roughly speaking, the objects (Definition 2.1) of $\mathscr{H}_{p}^{n}$ are homotopy classes of spaces endowed with certain structure maps, and the morphisms (Definition 2.3) are homotopy classes of pairs $(f, F)$, where $f$ is a map between objects and $F$ is a homotopy making the requisite diagram commute up to homotopy (Definition 2.2).

First we recall the May-Milgram [Ma, Mg1] combinatorial model for $\Omega^{n} \Sigma^{n} X$. Let $\mathbf{C}_{n}(q)$ denote Boardman and Vogt's [BV, Ma] space of ordered $q$-tuples of little cubes disjointly imbedded in $I^{n}$. The symmetric group $\Sigma_{q}$ acts freely on $\mathrm{C}_{n}(q)$ by permuting cubes. For a given space $X, C_{n} X$ is defined by

$$
C_{n} X=\left(\coprod_{q \geq 1} \mathbf{C}_{n}(q) \times_{\Sigma_{q}} X^{q}\right) / \sim
$$

where the equivalence relation is generated by $\left[\left(c_{1}, \ldots, c_{q}\right),\left(x_{1}, \ldots, x_{q-1}, *\right)\right]$ $\sim\left[\left(c_{1}, \ldots, c_{q-1}\right),\left(x_{1}, \ldots, x_{q-1}\right)\right]$, and $\Sigma_{q}$ acts on $X^{q}$ by permuting coordinates. There is a map

$$
\alpha_{n}: C_{n} X \rightarrow \Omega^{n} \Sigma^{n} X
$$

which is a weak homotopy equivalence when $X$ is path connected and preserves all additive structure.

The space $C_{n} X$ has a filtration in the $q$ variable; for $1 \leq p \leq \infty$ define $C_{n, p} X$ by

$$
C_{n, p} X=\left(\coprod_{1 \leq q \leq p} \mathbf{C}_{n}(q) \times \Sigma_{\Sigma_{q}} X^{q}\right) / \sim .
$$

Since $C_{n, p}$ is only defined for $n \geq 1$, we will adopt the convention that $C_{0, p} X=X$ for all $p$. This allows us to define the category $\mathscr{H}_{p}^{0}$, and it will correspond to the usual homotopy category of spaces (with no additional structure). There is a canonical "inclusion" of $X$ into $C_{n, p} X$ given by $x \mapsto[1, x]$, where by 1 here we mean the identity map $I^{n} \rightarrow I^{n}$. Via $\alpha_{n}$, this inclusion is compatible with the usual map $X \rightarrow \Omega^{n} \Sigma^{n} X$.

We are now in a position to define the objects of $\mathscr{H}_{p}^{n}$.

Definition 2.1. An $H_{p}^{n}$-space is a pair $\left(X, \theta_{n}^{X}\right)$, where $X$ is a space and $\theta_{n}^{X}$ is a (structure) map

$$
\theta_{n}^{X}: C_{n, p} X \rightarrow X
$$


which satisfies the property that the composition

$$
X \hookrightarrow C_{n, p} X \stackrel{\theta_{n}^{X}}{\longrightarrow} X
$$

is equal to the identity map.

We warn the reader that the definition of an $H_{p}^{n}$-space varies somewhat throughout the literature. For example, our $H_{2}^{n}$-space is equivalent to the $H_{n-1^{-}}$ space of Araki and Kudo [AK] (which also appears in [S11]); our $H_{p}^{n}$-space is called a "special $H_{(p)}^{(n-1)}$-space" by Dyer and Lashof [DL]. An H-space in our definition corresponds to an $H_{2}^{1}$-space.

In order to define the morphisms in $\mathscr{H}_{p}^{n}$, we first define the notion of an $H_{p}^{n}$-function.

Definition 2.2. Let $\left(X, \theta_{n}^{X}\right),\left(y, \theta_{n}^{Y}\right) \in \mathrm{Ob} \mathscr{H}_{p}^{n}$. An $H_{p}^{n}$-function from $X$ to $Y$ is a pair of maps $(f, F)$

$$
f: X \rightarrow Y, \quad F: C_{n, p} X \wedge I_{+} \rightarrow Y
$$

satisfying the properties

$$
\begin{array}{r}
F_{0}=f \circ \theta_{n}^{X}, \quad F_{1}=\theta_{n}^{Y} \circ C_{n, p} f, \\
F_{t}([1, x])=f(x) \quad \forall x \in X .
\end{array}
$$

Thus an $H_{p}^{n}$-function is a map $f$ between $H_{p}^{n}$-spaces together with a choice of homotopy $F$ making the following diagram homotopy commute:

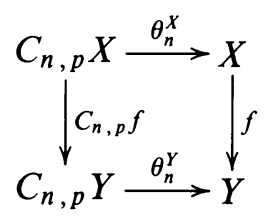

such that $F$ restricted to $X \wedge I_{+}$is the constant homotopy at $f$.

There is a composition law for $H_{p}^{n}$-functions defined as follows. Given $(f, F)$ from $X$ to $Y$ and $(g, G)$ from $Y$ to $Z$, define $(h, H)$ from $X$ to $Z$ by the formulas

$$
h=g \circ f, \quad H_{t}= \begin{cases}g \circ F_{2 t} & \text { if } t \in\left[0, \frac{1}{2}\right] ; \\ G_{2 t-1} \circ C_{n, p} f & \text { if } t \in\left[\frac{1}{2}, 1\right] .\end{cases}
$$

Note that this composition is not associative in general (although it is associative "up to homotopy" in a sense that will soon be made precise).

We now define an equivalence relation on $H_{p}^{n}$-functions. Suppose $(f, F)$ and $\left(f^{\prime}, F^{\prime}\right)$ are $H_{P}^{n}$-functions from $X$ to $Y$. Then $(f, F) \sim\left(f^{\prime}, F^{\prime}\right)$ if there is a pair $(P, Q)$ of homotopies

$$
\begin{gathered}
P: X \wedge I_{+} \rightarrow Y, \\
Q: C_{n, p} X \wedge I_{+} \wedge I_{+} \rightarrow Y
\end{gathered}
$$

satisfying the properties

$$
\begin{gathered}
P_{0}=f, \quad P_{1}=f^{\prime}, \\
Q_{s, 0}=F_{s}, \quad Q_{s, 1}=F_{s}^{\prime}, \\
Q_{0, t}=P_{t} \circ \theta_{n}^{X}, \quad Q_{1, t}=\theta_{n}^{Y} \circ C_{n, p} P_{t}, \\
Q_{s, t}([1, x])=P_{t}(x) .
\end{gathered}
$$


It is straightforward to verify that this is indeed an equivalence relation. Denote the equivalence class of $(f, F)$ by $[f, F]$, We are now ready to define the morphisms in $\mathscr{H}_{p}^{n}$.

Definition 2.3. An $H_{p}^{n}$-map (or $H_{p}^{n}$-morphism) is an equivalence class $[f, F]$ of $H_{p}^{n}$-functions. The composition law is given by $[g, G] \circ[f, F]=[h, H]$, where $(h, H)$ is the composition $(g, G) \circ(f, F)$ of $H_{p}^{n}$-functions.

Of course one must check in Definition 2.3 that the composition is well defined and associative and that ( $\mathrm{id}_{X}$, constant homotopy) is the identity for $X \in \mathrm{Ob} \mathscr{H}_{p}^{n}$. Verification of these is routine; e.g. associativity follows from the fact that stacking homotopies is associative up to homotopy, something verified when one shows the fundamental group is associative. Note also that the category $\mathscr{H}_{p}^{0}$ is just the usual homotopy category of spaces and that $\mathscr{H}_{2}^{1}$ is a homotopy category of $\mathrm{H}$-spaces.

Remark 2.4. A slightly more sophisticated way of viewing the category $\mathscr{H}_{p}^{n}$ is as follows. The set of $H_{p}^{n}$-functions from $X$ to $Y$ forms a subspace $H_{p}^{n}(X, Y)$ of $\operatorname{Map}(X, Y) \times \operatorname{Map}\left(C_{n, p} X \wedge I_{+}, Y\right)$, and the set of $H_{p}^{n}$-morphisms is just $\pi_{0}\left(H_{p}^{n}(X, Y)\right)$. Composition of $H_{p}^{n}$-functions induces homotopy associative continuous pairings

$$
H_{P}^{n}(Y, Z) \times H_{p}^{n}(X, Y) \rightarrow H_{p}^{n}(X, Z)
$$

which induce composition in $\mathscr{H}_{p}^{n}$ on the path components.

\section{THE FUnCTORS $\Omega^{n}$ AND $P_{p}^{n}$}

The purpose of this section is to define functors $\Omega^{n}: \mathscr{H}_{p}{ }^{k} \rightarrow \mathscr{H}_{p}{ }^{n+k}$ (Definitions 3.1, 3.2, and 3.3) and $P_{p}^{n}: \mathscr{H}_{p}^{n} \rightarrow \mathscr{H}_{p}^{0}$ (Definitions 3.4, 3.5, and 3.6) for $1 \leq n<\infty$. The functor $\Omega^{n}$, which is the $n$-fold loop space functor, is of course well known. However, in later sections we will need a good definition of $\Omega^{n}$ in order to prove the adjointness of $\Omega^{n}$ and $P_{p}^{n}$. The reader should note for future reference that we are most interested in $\Omega^{n}$ above when $k=0$; this is the one that will be the right adjoint to $P_{p}^{n}$. At the end of this section, we give a characterization (Proposition 3.7 and Corollary 3.8) of our map $h$ which will be useful in comparing our constructions with the usual Hopf construction.

In [Ma] (Proposition 5.4), a natural transformation $\Sigma^{n} C_{n+k, p} \rightarrow C_{k, p} \Sigma^{n}$ is defined. Denote this natural transformation by $\varepsilon_{n}$. Let $\epsilon: \Sigma^{n} \Omega^{n} \rightarrow 1$ be the natural transformation given by evaluation. Finally, let $\alpha_{n}: C_{n, p} \rightarrow \Omega^{n} \Sigma^{n}$ be the natural transformation coming from (2.2). The map $\varepsilon_{n}$ fits into the following naturally commutative diagram:

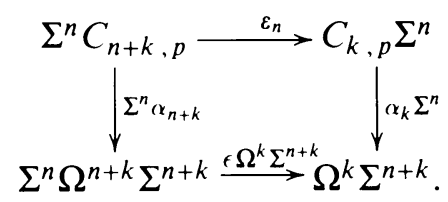

Thus one should think of $\varepsilon_{n}$ as an "evaluation" map that sits over $\epsilon$. Denote the adjoint of $\varepsilon_{n}$ by $\hat{\varepsilon}_{n}: C_{n+k, p} \rightarrow \Omega^{n} C_{k, p} \Sigma^{n}$; note that when $k=0, \hat{\varepsilon}_{n}=\alpha_{n}$. In $\S 4$ we will need the following compatibility properties of $\epsilon, \varepsilon_{n}$ and $\hat{\varepsilon}_{n}$ :

$$
\epsilon \circ \Sigma^{n} \hat{\varepsilon}_{n}=\varepsilon_{n}, \quad \Sigma^{k} \epsilon \circ \varepsilon_{n+k}=\varepsilon_{k} \circ \Sigma^{k} C_{k, p} \epsilon \circ \varepsilon_{n} .
$$


Definition 3.1. Let $\left(X, \theta_{k}^{X}\right) \in \mathrm{Ob} \mathscr{H}_{p}^{k}$. The functor $\Omega^{n}: \mathscr{H}_{p}^{k} \rightarrow \mathscr{H}_{p}^{n+k}$ is defined on objects by the formula

$$
\Omega^{n}\left(X, \theta_{k}^{X}\right)=\left(\Omega^{n} X, \theta_{n+k}^{\Omega^{n} X}\right),
$$

where $\theta_{n+k}^{\Omega^{n} X}$ is the composite

$$
C_{n+k, p} \Omega^{n} X \stackrel{\hat{\varepsilon}_{n}}{\longrightarrow} \Omega^{n} C_{k, p} \Sigma^{n} \Omega^{n} X \stackrel{\Omega^{n} C_{k, p} \epsilon}{\longrightarrow} \Omega^{n} C_{k, p} X \stackrel{\Omega^{n} \theta_{k}^{X}}{\longrightarrow} \Omega^{n} X .
$$

Because the composite

$$
\Omega^{n} X \hookrightarrow C_{n+k, p} \Omega^{n} X \stackrel{\hat{\varepsilon}_{n}}{\longrightarrow} \Omega^{n} C_{k, p} \Sigma^{n} \Omega^{n} X \stackrel{\Omega^{n} C_{k, p} \epsilon}{\longrightarrow} \Omega^{n} C_{k, p} X
$$

is equal to $\Omega^{n}$ applied to $X \hookrightarrow C_{k, p} X$, and $\Omega^{n}$ takes the identity map to the identity map, this definition really does make $\Omega^{n} X$ into an $H_{p}^{n}$-space.

In order to define $\Omega^{n}$ on morphisms, we first define it on $H_{p}^{k}$-functions.

Definition 3.2. Let $(f, F)$ be an $H_{p}^{k}$-function. The $H_{p}^{n+k}$-function $\Omega^{n}(f, F)$ is defined by the formula

$$
\Omega^{n}(f, F)=\left(\Omega^{n} f, \bar{\Omega}^{n} F\right),
$$

where

$$
\left(\bar{\Omega}^{n} F\right)_{t}=\Omega^{n} F_{t} \circ \Omega^{n} C_{k, p} \epsilon \circ \hat{\varepsilon}_{n} .
$$

Now if $(f, F) \sim\left(f^{\prime}, F^{\prime}\right)$ with homotopies $(P, Q)$, then it is not difficult to see that $\Omega^{n}(f, F) \sim \Omega^{n}\left(f^{\prime}, F^{\prime}\right)$ with homotopies $\left(\Omega^{n} P, \bar{\Omega}^{n} Q\right)$, where by definition

$$
\left(\Omega^{n} P\right)_{t}=\Omega^{n} P_{t}, \quad\left(\bar{\Omega}^{n} Q\right)_{s, t}=\Omega^{n} Q_{s, t} \circ \Omega^{n} C_{k, p} \epsilon \circ \hat{\varepsilon}_{n} .
$$

Thus we may define $\Omega^{n}$ on $[f, F] \in$ Mor $\mathscr{H}_{p}^{k}$ as follows.

Definition 3.3. Let $[f, F] \in \operatorname{Mor} \mathscr{H}_{p}{ }^{k}$. The functor $\Omega^{n}: \mathscr{H}_{p}{ }^{k} \rightarrow \mathscr{H}_{p}{ }^{n+k}$ is defined on morphisms by the formula

$$
\Omega^{n}[f, F]=\left[\Omega^{n} f, \bar{\Omega}^{n} F\right],
$$

where $\bar{\Omega}^{n}$ is given in Definition 3.2.

The functor $P_{p}^{n}$ requires somewhat more care to define. Recall the "inclusion" mapping $X \hookrightarrow C_{n, p} X$ from $\S 2$. Let

$$
\widetilde{C}_{n, p} X=C_{n, p} X \cup_{X}(X \wedge I)
$$

i.e. $\widetilde{C}_{n, p} X$ is the (reduced) mapping cone of $X \hookrightarrow C_{n, p} X$ (we adopt the convention that $I=[0,1]$ with basepoint 1). One should think of $\widetilde{C}_{n, p} X$ as a "reduced" version of $C_{n, p} X$. Consider the structure map $\theta_{n}^{X}: C_{n, p} X \rightarrow X$. This map will be used to define a "reduced" structure map

$$
h: \Sigma^{n} \widetilde{C}_{n, p} X \rightarrow \Sigma^{n} X .
$$

Note that $\Sigma^{n} \widetilde{C}_{n, p} X=\Sigma^{n} C_{n, p} X \cup_{\Sigma^{n} X}\left(\Sigma^{n} X \wedge I\right)$. 
Deiinition 3.4. Suppose $n \geq 1$ and let $\left(X, \theta_{n}^{X}\right) \in \mathrm{Ob} \mathscr{H}_{p}{ }^{n}$. The map $h: \Sigma^{n} \widetilde{C}_{n, p} X$ $\rightarrow \Sigma^{n} X$ associated to $\left(X, \theta_{n}^{X}\right)$ is defined on $\Sigma^{n} C_{n, p} X$ as $\Sigma^{n} \theta_{n}^{X}-\varepsilon_{n}$, and on $\Sigma^{n} X \wedge I$ as the homotopy from $\operatorname{id}_{\Sigma^{n} X}-\operatorname{id}_{\Sigma^{n} X}$ to the constant map given by the formula

$$
h(u, v, x, s)= \begin{cases}(2 u(1-s), v, x) & \text { if } u \in\left[0, \frac{1}{2}\right] \\ (2(1-u)(1-s), v, x) & \text { if } u \in\left[\frac{1}{2}, 1\right],\end{cases}
$$

where the point $(u, v, x, s)$ above is considered as an element of $S^{1} \wedge S^{n-1} \wedge$ $X \wedge I=\Sigma^{n} X \wedge I$. Here "- " means use the first suspension coordinate to subtract maps; i.e. if $\alpha, \beta: \Sigma Y \rightarrow Z$, then $\alpha-\beta: \Sigma Y \rightarrow Z$ is given by the formula

$$
(\alpha-\beta)(u, y)= \begin{cases}\alpha(2 u, y) & \text { if } u \in\left[0, \frac{1}{2}\right] ; \\ \beta(2(1-u), y) & \text { if } u \in\left[\frac{1}{2}, 1\right] .\end{cases}
$$

Since $\Sigma^{n} \theta_{n}^{X}$ and $\varepsilon_{n}$ restricted to $\Sigma^{n} X$ both give the identity map, the definition of $h$ agrees on the overlap of the two domains above.

In Corollary 3.8 below and in Appendix A we verify that when $n=1$ and $p=2$ this corresponds, after one suspension, to the usual Hopf construction for an H-space $X$. We also note here that when $p=2$ and $n>1$, this map is defined and used along with Adams spectral sequence techniques in [Mg2].

We are now in position to define $P_{p}^{n}$ on objects.

Definition 3.5. Let $\left(X, \theta_{n}^{X}\right) \in \mathrm{Ob} \mathscr{H}_{p}{ }^{n}$. The functor $P_{p}^{n}: \mathscr{H}_{p}^{n} \rightarrow \mathscr{H}_{p}^{0}$ is defined on objects for $n=0$ as $P_{p}^{0} X=X$, and for $n \geq 1$ as the (reduced) mapping cone of $h$; that is,

$$
P_{p}^{n} X=\Sigma^{n} X \cup_{h}\left(\Sigma^{n} \widetilde{C}_{n, p} X \wedge I\right) .
$$

The definition of $P_{p}^{n}$ on morphisms also requires some preliminary discussion. Suppose $[f, F] \in \operatorname{Mor} \mathscr{H}_{p}^{n}$ is a morphism from $X$ to $Y$. Let $(f, F)$ be a representative $H_{P}^{n}$-function. As in the construction of $P_{p}^{n}$ (for $\left.n \geq 1\right)$ on objects, we need a "reduced" version of $F$. Let

$$
\widetilde{F}: \Sigma^{n} \widetilde{C}_{n, p} X \wedge I_{+} \rightarrow \Sigma^{n} Y
$$

be defined as follows. On $\Sigma^{n} C_{n, p} X \wedge I_{+}$, let $\widetilde{F}_{t}$ be defined as the map $\Sigma^{n} F_{t}-$ $\left(\Sigma^{n} f \circ \varepsilon_{n}\right)$. And on $\Sigma^{n} X \wedge I \wedge I_{+}$, let $\widetilde{F}_{t}$ be defined (for all $t$ ) as the homotopy $\Sigma^{n} f \circ h$ from $\Sigma^{n} f-\Sigma^{n} f$ to the constant map. The reader can verify that the definition of $\widetilde{F}$ agrees on the overlaps of the domains above (using the fact that $\left.\Sigma^{n} f \circ \varepsilon_{n}=\varepsilon_{n} \circ \Sigma^{n} C_{n, p} f\right)$, and that $\widetilde{F}$ makes the following diagram commute up to homotopy:

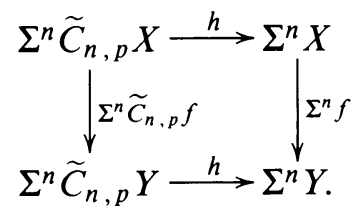

In particular, $\widetilde{F}_{0}=\Sigma^{n} f \circ h$ and $\widetilde{F}_{1}=h \circ \Sigma^{n} \widetilde{C}_{n, p} f$. We can now define $P_{p}^{n}$ on morphisms. 
Defintion 3.6. Let $[f, F] \in \operatorname{Mor} \mathscr{H}_{p}^{n}$. The functor $P_{p}^{n}: \mathscr{H}_{p}^{n} \rightarrow \mathscr{H}_{p}^{0}$ is defined on morphisms as the homotopy class of the following map. For $n=0, P_{p}^{0}[f, F]=$ $f$, and for $n \geq 1, P_{p}^{n}[f, F]$ is defined on $\Sigma^{n} X$ as $\Sigma^{n} f$, and on $\Sigma^{n} \widetilde{C}_{n, p} X \wedge I$ by the formula

$$
P_{p}^{n}[f, F](a, t)= \begin{cases}\widetilde{F}_{2 t}(a) & \text { if } t \in\left[0, \frac{1}{2}\right] \\ \left(\Sigma^{n} \widetilde{C}_{n, p} f(a), 2 t-1\right) & \text { if } t \in\left[\frac{1}{2}, f\right]\end{cases}
$$

for each point $a \in \Sigma^{n} \widetilde{C}_{n, p} X$.

In the definition above, when $t \in\left[0, \frac{1}{2}\right]$ the range of $P_{p}^{n}[f, F]$ is $\Sigma^{n} Y$, and when $t \in\left[\frac{1}{2}, 1\right]$ the range is $\Sigma^{n} \widetilde{C}_{n, p} Y \wedge I$. The reader can verify that these maps agree on the overlaps of their domains. Of course, Definition 3.6 as stated above depends on the choice of the representative $H_{p}^{n}$-function for the morphism $[f, F]$. However, an equivalence between $H_{p}^{n}$-functions $(f, F)$ and $\left(f, F^{\prime}\right)$ gives rise to a homotopy $P_{p}^{n}[f, F] \simeq P_{p}^{n}\left[f, F^{\prime}\right]$ in the obvious way. Thus Definition 3.6 really does not depend on the choice of representative $H_{p}^{n}$-function for $[f, F]$. Also, if $[g, G]$ is a morphism from $Y$ to $Z$, it is straightforward to verify that the homotopy class of $P_{p}^{n}[g, G] \circ P_{p}^{n}[f, F]$ is the same as that of $P_{p}^{n}([g, G] \circ[f, F])$. Finally, it is easy to see that $P_{p}^{n}$ applied to the identity morphism is homotopic to the identity map. Thus we get a well-defined functor $P_{p}^{n}$.

Since $P_{p}^{n}$ was defined on objects as the mapping cone of $h$, there is a cofibration sequence (for $n \geq 1$ )

$$
\Sigma^{n} \widetilde{C}_{n, p} X \stackrel{h}{\longrightarrow} \Sigma^{n} X \stackrel{i}{\rightarrow} P_{p}^{n} X \stackrel{j}{\longrightarrow} \Sigma^{n+1} \widetilde{C}_{n, p} X,
$$

where the maps $h, i$ and $j$ are natural transformations of functors from $\mathscr{H}_{p}^{n}$ to $\mathscr{H}_{p}^{0}$. Henceforth this will be called the basic cofibration sequence. This sequence will be exploited in $\S 6$ when we consider the computation of $\widetilde{H}^{*}\left(P_{2}^{n} X ; \mathbf{F}_{2}\right)$.

We end this section with a useful characterization of $h$, which will be used in Appendix $\mathrm{A}$ in the discussion about how our construction compares with the usual Hopf construction when $n=1$ and $p=2$.

Proposition 3.7. Let $\left(X, \theta_{n}^{X}\right) \in \mathrm{Ob} \mathscr{H}_{p}^{n}$ with $n \geq 1$. Then up to homotopy, the map $h$ is the unique map making the diagram

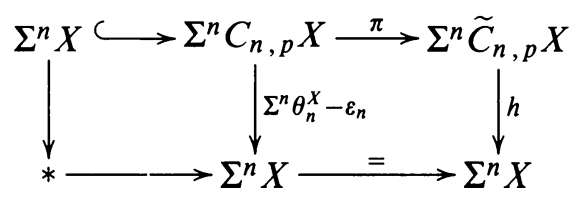

homotopy commute.

Proof. The map $\pi$ admits a homotopy section for any $X$. Q.E.D.

If $\left(X, \theta_{1}^{X}\right) \in \mathrm{Ob} \mathscr{C}_{2}^{1}$, let $\theta: X \times X \rightarrow X$ be the composite $X \times X \rightarrow$ $C_{1,2} X \stackrel{\theta_{1}^{X}}{\longrightarrow} X$, i.e. the H-space multiplication map. Note that $\Sigma \widetilde{C}_{1,2} X \simeq$ $\Sigma(X \wedge X)$. 
Corollary 3.8. Up to homotopy, $h$ is the unique map making the diagram

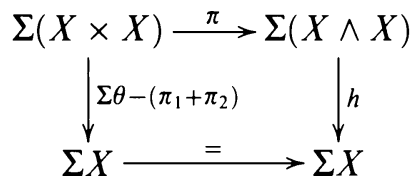

homotopy commute.

Proof. By inspecting the definition of $\varepsilon_{1}: \Sigma C_{1,2} X \rightarrow \Sigma X$, one sees that the map $X \times X \rightarrow C_{1,2} X$ can be chosen so that

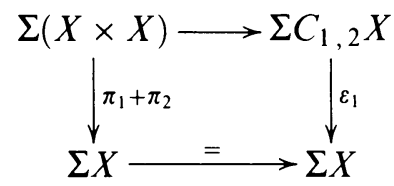

commutes (where the $\pi_{i}$ are the obvious projection maps). Therefore we obtain a homotopy commutative diagram

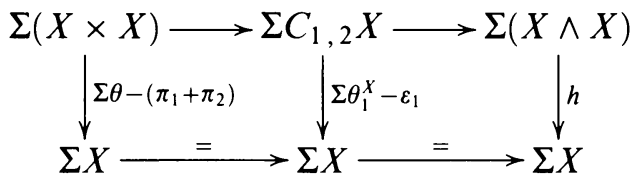

where the top horizontal composite is $\pi$. Q.E.D.

There are actually two obvious choices (up to homotopy) for the map $X \times$ $X \rightarrow C_{1,2} X$; the second choice can be characterized by the truth of Corollary 3.8 with $\pi_{1}+\pi_{2}$ replaced by $\pi_{2}+\pi_{1}$. Thus there are actually two (essentially equivalent) versions of Corollary 3.8. This observation is useful in understanding the comparison between $h$ and the usual Hopf construction in Appendix A.

\section{The ADJOINTNesS of $\Omega^{n}$ AND $P_{p}^{n}$}

The purpose of this section is to show that the functor $P_{p}^{n}: \mathscr{H}_{p}^{n} \rightarrow \mathscr{H}_{p}^{0}$ is left adjoint to $\Omega^{n}: \mathscr{H}_{p}^{0} \rightarrow \mathscr{H}_{p}^{n}$ (Theorem 4.9), just as is $\Sigma^{n}$ (resp. the $n$th classifying space, $B^{n}$ ), considered as a functor from the category $\mathscr{H}_{p}^{0}$ (resp. the homotopy category of $n$-fold loop spaces) to $\mathscr{H}_{p}^{0}$. Of course, here we are thinking of $\Omega^{n}$ as taking its values in three different categories

$$
n \text {-fold loop spaces } \subset \mathscr{H}_{p}^{n} \subset \mathscr{H}_{p}^{0} \text {. }
$$

Thus one should think of $P_{p}^{n}$ as lying "between" $\Sigma^{n}$ and $B^{n}$ (keeping in mind that $B^{n} \Omega^{n}=1$ ). In fact, this will be made precise in the course of proving the adjointness of $\Omega^{n}$ and $P_{p}^{n}$. Also, in the course of the proof we will demonstrate the compatibility properties of the maps $h$ with our various evaluation maps (Theorem 4.1).

In order to prove the adjointness of $\Omega^{n}$ and $P_{p}^{n}$, natural transformations

$$
\epsilon: P_{p}^{n} \Omega^{n} \rightarrow 1, \quad \eta: 1 \rightarrow \Omega^{n} P_{p}^{n}
$$

are constructed (Definitions 4.2 and 4.5, and Theorems 4.3 and 4.6) and shown to have the usual universal properties (Theorems 4.7 and 4.8). 
Consider first $\epsilon: P_{p}^{n} \Omega^{n} \rightarrow 1$. One property that $\epsilon$ must have in order to make sense is that it must be an extension of the usual evaluation $\epsilon: \Sigma^{n} \Omega^{n} \rightarrow 1$; i.e. the following diagram of natural transformations should commute:

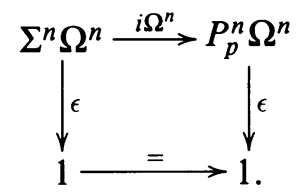

In fact, we will define a slightly more general $\epsilon: P_{p}^{n+k} \Omega^{n} \rightarrow P_{p}^{k}$ which makes the following diagram commute:

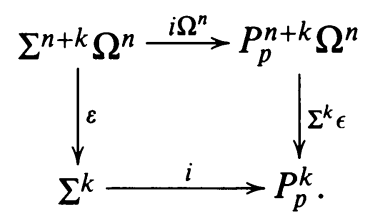

For this the following theorem is needed.

Theorem 4.1. (i) Suppose $k \geq 1$, and let $\left(X, \theta_{k}^{X}\right) \in \mathrm{Ob} \mathscr{H}_{p}^{k}$. Then the constructions for $\left(X, \theta_{k}^{X}\right)$ and $\left(\Omega^{n} X, \theta_{n+k}^{\Omega^{n} X}\right) \in \mathrm{Ob} \mathscr{H}_{p}{ }^{n+k}$ fit into the following naturally (strictly) commutative diagram:

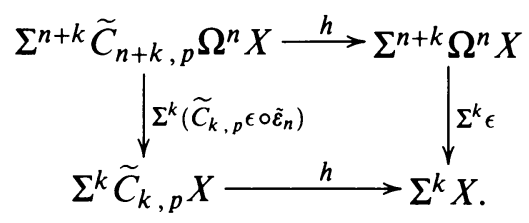

(ii) If $X \in \mathrm{Ob} \mathscr{H}_{p}^{0}$, then for $\left(\Omega X, \theta_{1}^{\Omega X}\right) \in \mathrm{Ob} \mathscr{H}_{p}^{1}$, the following compcisite is naturally null-homotopic:

$$
\Sigma \widetilde{C}_{1, p} \Omega X \stackrel{h}{\longrightarrow} \Sigma \Omega X \stackrel{\epsilon}{\rightarrow} X .
$$

Proof. Before proving the theorem, the definition of $\tilde{\varepsilon}_{n}$ needs to be given. Recall the natural transformation $\varepsilon_{n}: \Sigma^{n} C_{n+k, p} \rightarrow C_{k, p} \Sigma^{n}$ from $\S 3$. Define the natural transformation $\tilde{\varepsilon}_{n}: \Sigma^{n} \widetilde{C}_{n+k, p} \rightarrow \tilde{C}_{k, p} \Sigma^{n}$ to be $\varepsilon_{n}$ on $\Sigma^{n} C_{n+k, p} X$ and the identity on $\Sigma^{n} X \wedge I$.

For (i), in order to show that the diagram commutes, it suffices to check that it commutes on the various pieces.

On $\Sigma^{n+k} C_{n+k, p} \Omega^{n} X$

$$
\begin{aligned}
\Sigma^{k} \epsilon \circ h & =\Sigma^{k} \epsilon \circ\left(\Sigma^{n+k} \theta_{n+k}^{\Omega^{n} X}-\varepsilon_{n+k}\right) \\
& =\Sigma^{k}\left(\epsilon \circ \Sigma^{n} \Omega^{n} \theta_{k}^{X} \circ \Sigma^{n} \Omega^{n} C_{k, p} \epsilon \circ \Sigma^{n} \hat{\varepsilon}_{n}\right)-\left(\Sigma^{k} \epsilon \circ \varepsilon_{n+k}\right) \\
& =\Sigma^{k}\left(\theta_{k}^{X} \circ C_{k, p} \epsilon \circ \epsilon \circ \Sigma^{n} \hat{\varepsilon}_{n}\right)-\left(\Sigma^{k} \epsilon \circ \varepsilon_{n+k}\right) \\
& =\Sigma^{k}\left(\theta_{k}^{X} \circ C_{k, p} \epsilon \circ \varepsilon_{n}\right)-\left(\varepsilon_{k} \circ \Sigma^{k} C_{k, p} \epsilon \circ \varepsilon_{n}\right) \\
& =h \circ \Sigma^{k}\left(\widetilde{C}_{k, p} \epsilon \circ \tilde{\varepsilon}_{n}\right) .
\end{aligned}
$$

Here we use the compatibility properties from (3.2)

$$
\epsilon \circ \Sigma^{n} \hat{\varepsilon}_{n}=\varepsilon_{n}, \quad \Sigma^{k} \epsilon \circ \varepsilon_{n+k}=\varepsilon_{k} \circ \Sigma^{k} C_{k, p} \epsilon \circ \varepsilon_{n} .
$$


On $\Sigma^{n+k} \Omega^{n} X \wedge I$,

$$
\Sigma^{k} \epsilon \circ h=h \circ \Sigma^{k} \epsilon=h \circ \Sigma^{k}\left(\widetilde{C}_{k, p} \epsilon \circ \tilde{\varepsilon}_{n}\right) .
$$

In this case we use the fact that $k \geq 1$ (this is essential to the proof) and $h$ is the null-homotopy of $\operatorname{id}_{\Sigma^{k} X}-\mathrm{id}_{\Sigma^{k} X}$, where the first suspension coordinate is used to subtract maps, so that $h$ commutes with the evaluation. This completes the proof of (i).

For (ii), on $\Sigma C_{1, p} \Omega X$,

$$
\begin{aligned}
\epsilon \circ h & =\epsilon \circ\left(\Sigma \theta_{1}^{\Omega X}-\varepsilon_{1}\right) \\
& =\left(\epsilon \circ \Sigma \Omega \theta_{0}^{X} \circ \Sigma \Omega C_{0, p} \epsilon \circ \Sigma \hat{\varepsilon}_{1}\right)-\left(\epsilon \circ \varepsilon_{1}\right) \\
& =\left(\epsilon \circ \epsilon \circ \Sigma \hat{\varepsilon}_{1}\right)-\left(\epsilon \circ \varepsilon_{1}\right) \\
& =\left(\epsilon \circ \varepsilon_{1}\right)-\left(\epsilon \circ \varepsilon_{1}\right) .
\end{aligned}
$$

On $\Sigma \Omega X \wedge I, \epsilon \circ h$ is the usual null-homotopy (see (3.12)) of $\epsilon-\epsilon$.

Define a null-homotopy $N: \Sigma \widetilde{C}_{1, p} \Omega X \wedge I_{+} \rightarrow X$ by the following formulas. On $\Sigma C_{1, p} \Omega X \wedge I_{+}$, for $(u,[c, \lambda]) \in S^{1} \wedge C_{1, p} \Omega X$, let

$$
N_{t}(u,[c, \lambda])= \begin{cases}\epsilon \circ \varepsilon_{1}(2 u(1-t),[c, \lambda]) & \text { if } u \in\left[0, \frac{1}{2}\right] ; \\ \epsilon \circ \varepsilon_{1}(2(1-u)(1-t),[c, \lambda]) & \text { if } u \in\left[\frac{1}{2}, 1\right] .\end{cases}
$$

On $\Sigma \Omega X \wedge I \wedge I_{+}$, for $(u, \lambda, s) \in S^{1} \wedge \Omega X \wedge I$, let

$$
N_{t}(u, \lambda, s)= \begin{cases}\epsilon(2 u(1-s-t), \lambda) & \text { if } u \in\left[0, \frac{1}{2}\right] \\ \epsilon(2(1-u)(1-s-t), \lambda) & \text { if } u \in\left[\frac{1}{2}, 1\right] .\end{cases}
$$

One can easily check that $N$ is continuous and is indeed a null-homotopy of $\epsilon \circ h$. Q.E.D.

Using Theorem 4.1 we can make the following definition.

Definition 4.2. Let $k \geq 0$ and suppose $\left(X, \theta_{k}^{X}\right) \in \mathrm{Ob} \mathscr{H}_{p}^{k}$. The map

$$
\epsilon: P_{p}^{n+k} \Omega^{n} X \rightarrow P_{p}^{k} X
$$

is defined for $k \geq 1$ as the induced map on mapping cones from the diagram of part (i) of Theorem 4.1, and for $k=0$ as the composite mapping

$$
P_{p}^{n} \Omega^{n} X \rightarrow P_{p}^{1} \Omega X \rightarrow X,
$$

where the first map is the one induced by part (i) of Theorem 4.1 and the second map is the one induced by the null-homotopy $N$ from part (ii) of Theorem 4.1.

This definition clearly makes diagram (4.4) commutative. The next theorem insures that this definition of $\epsilon$ is also natural.

Theorem 4.3. Let $\epsilon: P_{p}^{n+k} \Omega^{n} \rightarrow P_{p}^{k}$ be the map from Definition 4.2. Then $\epsilon$ is a natural transformation of functors.

Proof. Suppose $\left(X, \theta_{k}^{X}\right),\left(Y, \theta_{k}^{Y}\right) \in \mathrm{Ob} \mathscr{H}_{p}^{k}$ and let $[f, F]$ be a morphism from $X$ to $Y$. Then it suffices to show that the following diagram commutes in the category $\mathscr{H}_{p}^{0}$ (i.e. commutes up to homotopy):

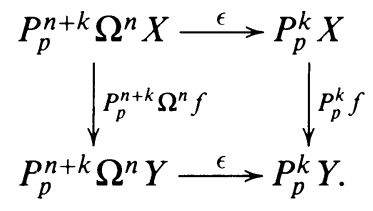


This is first shown in the case that $k \geq 1$. In this case it will be shown that the diagram above actually strictly commutes. In view of the commutative diagram (4.5) of Theorem 4.1, the naturality of the maps $\varepsilon_{n}, \Sigma^{k}\left(\widetilde{C}_{k, p} \epsilon \circ \tilde{\varepsilon}_{n}\right)$, and $\Sigma^{k} \epsilon$, the definition of $h$, and the construction of $P_{p}^{n+k} \Omega^{n}$ and $P_{p}^{k}$ on morphisms, it suffices to show that the following diagram commutes:

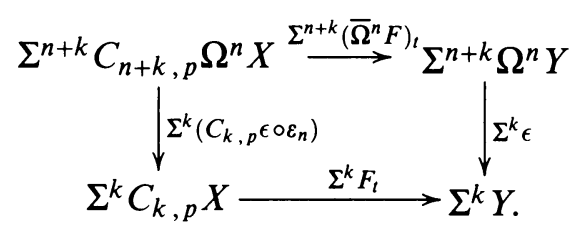

Checking the definitions and using (3.2), one sees that

$$
\begin{aligned}
\Sigma^{k} \epsilon \circ \Sigma^{n+k}\left(\bar{\Omega}^{n} F\right)_{t} & =\Sigma^{k} \epsilon \circ \Sigma^{n+k}\left(\Omega^{n} F_{t} \circ \Omega^{n} C_{k, p} \epsilon \circ \hat{\varepsilon}_{n}\right) \\
& =\Sigma^{k} F_{t} \circ \Sigma^{k}\left(C_{k, p} \epsilon \circ \odot \circ \Sigma^{n} \hat{\varepsilon}_{n}\right) \\
& =\Sigma^{k} F_{t} \circ \Sigma^{k}\left(C_{k, p} \epsilon \circ \varepsilon_{n}\right) .
\end{aligned}
$$

This completes the proof in the case that $k \geq 1$.

Now suppose that $k=0$. In this case the diagram in question is

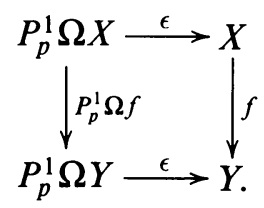

Because $\epsilon: \Sigma \Omega \rightarrow 1$ and the null-homotopy $N$ from Theorem 4.1 (ii) are both natural with respect to maps, the diagram above will commute up to homotopy if we can show that the following diagram commutes:

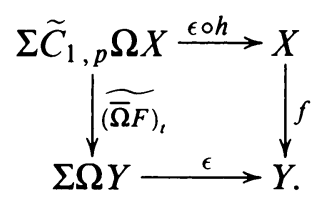

Checking the definitions, using the fact that in this case $F_{t}=f$, and again using (3.2), one sees that on $\Sigma C_{1, p} \Omega X$,

$$
\begin{aligned}
\epsilon \circ(\widetilde{\bar{\Omega} F})_{t} & =\epsilon \circ \Sigma\left(\left(\Omega F_{t} \circ \Omega \epsilon \circ \hat{\varepsilon}_{1}\right)-\left(\Omega f \circ \varepsilon_{1}\right)\right) \\
& =\left(F_{t} \circ \epsilon \circ \epsilon \circ \Sigma \hat{\varepsilon}_{1}\right)-\left(f \circ \epsilon \circ \varepsilon_{1}\right) \\
& =f \circ\left(\left(\epsilon \circ \varepsilon_{1}\right)-\left(\epsilon \circ \varepsilon_{1}\right)\right) \\
& =f \circ \epsilon \circ h ;
\end{aligned}
$$

and on $\Sigma X \wedge I$,

$$
\epsilon \circ(\widetilde{\bar{\Omega} F})_{t}=\epsilon \circ \Sigma \Omega f \circ h=f \circ \epsilon \circ h .
$$

This completes the proof. Q.E.D.

We now turn to the definition of $\eta: 1 \rightarrow \Omega^{n} P_{p}^{n}$. As was the case with $\epsilon, \eta$ is related to the usual natural transformation $\eta: 1 \rightarrow \Omega^{n} \Sigma^{n}$. The usual version 
of $\eta$ is defined as the adjoint of the identity $\Sigma^{n} \rightarrow \Sigma^{n}$. Thus our version of $\eta$ will be defined as the adjoint of $i: \Sigma^{n} \rightarrow P_{p}^{n}$. Of course, in order for $\eta$ to be a natural transformation of functors, the map $X \stackrel{\eta}{\longrightarrow} \Omega^{n} P_{p}^{n} X$ must be a morphism in the category $\mathscr{H}_{p}^{n}$ for each $\left(X, \theta_{n}^{X}\right) \in \mathrm{Ob} \mathscr{H}_{p}^{n}$. Thus we must find a (natural) homotopy

$$
\Phi: C_{n, p} X \wedge I_{+} \rightarrow \Omega^{n} P_{p}^{n} X
$$

which makes $(\eta, \Phi)$ into an $H_{p}^{n}$-function.

In order for $(\eta, \Phi)$ to be an $H_{p}^{n}$-function, $\Phi$ must satisfy

$$
\begin{gathered}
\Phi_{0}=\eta \circ \theta_{n}^{X}, \quad \Phi_{1}=\theta_{n}^{\Omega^{n} P_{p}^{n} X} \circ C_{n, p} \eta, \\
\Phi_{t}([1, x])=\eta(x) \quad \forall x \in X .
\end{gathered}
$$

Consider the adjoint situation; in this case

$$
\widehat{\Phi}: \Sigma^{n} C_{n, p} X \wedge I_{+} \rightarrow P_{p}^{n} X
$$

must satisfy

$$
\begin{gathered}
\widehat{\Phi}_{0}=i \circ \Sigma^{n} \theta_{n}^{X}, \quad \widehat{\Phi}_{1}=i \circ \varepsilon_{n}, \\
\widehat{\Phi}_{t}(w,[1, x])=i(w, x) \quad \forall(w, x) \in S^{n} \wedge X .
\end{gathered}
$$

The identity $\widehat{\Phi}_{1}=i \circ \varepsilon_{n}$ follows from commutativity of the diagram

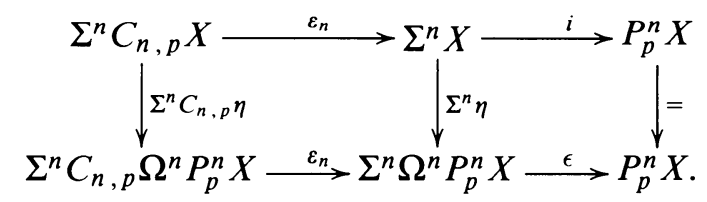

The left square commutes since $\varepsilon_{n}$ is natural, and the right square commutes since $\eta$ is adjoint to $i$. Checking the definitions, one can see that $\epsilon \circ \varepsilon_{n} \circ \Sigma^{n} C_{n, p} \eta$ is adjoint to $\theta_{n}^{\Omega^{n} P_{p}^{n} X} \circ C_{n, p} \eta$.

Of course $\Sigma^{n} \theta_{n}^{X}$ and $\varepsilon_{n}$ are not homotopic in general; however, the point of the construction of $P_{p}^{n} X$ is that $\left(i \circ \Sigma^{n} \theta_{n}^{X}\right)-\left(i \circ \varepsilon_{n}\right)=i \circ\left(\Sigma^{n} \theta_{n}^{X}-\varepsilon_{n}\right)$ is null-homotopic, so that $i \circ \Sigma^{n} \theta_{n}^{X}$ and $i \circ \varepsilon_{n}$ are homotopic. With a certain amount of care, we can construct this homotopy in a natural way.

Lemma 4.4. For $\left(X, \theta_{n}^{X}\right) \in \mathrm{Ob} \mathscr{H}_{p}^{n}$, there is a homotopy $\widehat{\Phi}: \Sigma^{n} C_{n, p} X \wedge I_{+} \rightarrow$ $P_{p}^{n} X$ which satisfies the properties

$$
\begin{gathered}
\widehat{\Phi}_{0}=i \circ \Sigma^{n} \theta_{n}^{X}, \quad \widehat{\Phi}_{1}=i \circ \varepsilon_{n}, \\
\widehat{\Phi}_{t}(w,[1, x])=i(w, x) \quad \forall(w, x) \in S^{n} \wedge X .
\end{gathered}
$$

Proof. For a point $(u, v,[c, x]) \in S^{1} \wedge S^{n-1} \wedge C_{n, p} X=\Sigma^{n} C_{n, p} X$, define a 
homotopy $\Psi: \Sigma^{n} C_{n, p} X \wedge I_{+} \rightarrow P_{p}^{n} X$ by the formula (4.28)

$$
\Psi_{t}(u, v,[c, x])= \begin{cases}1 .\left(\frac{3 u}{4-4 t}, v,[c, x], 2 t-1\right) & \text { if } t \in\left[\frac{1}{2}, 1\right], 4 t \leq 4-3 u \\ 2 \cdot \varepsilon_{n}\left(\frac{3 u-4+4 t}{4 t-1}, v,[c, x]\right) & \text { if } t \in\left[\frac{1}{2}, 1\right], 4-3 u \leq 4 t \\ 3 .\left(\frac{3 u}{3-4 t}, v, \theta_{n}^{X}[c, x]\right) & \text { if } t \in\left[0, \frac{1}{2}\right], 4 t \leq 3-3 u \\ 4 . \varepsilon_{n}(4-3 u-4 t, v,[c, x]) & \text { if } t \in\left[0, \frac{1}{2}\right], \\ 5 . \varepsilon_{n}(3 u-2, v,[c, x]) & 3-3 u \leq 4 t \leq 6-6 u\end{cases}
$$

A picture representing $\Psi$ is shown below. Noting that the horizontal direction is $u$ and the vertical direction is $t$, the labelled regions on the picture correspond to the labels above, which are the following maps.

1: Coning off of $i \circ\left(\Sigma^{n} \theta_{n}^{X}-\varepsilon_{n}\right)$ within $\Sigma^{n} \widetilde{C}_{n, p} X \wedge I$.

2: The constant homotopy at $i \circ \varepsilon_{n}$.

3: The constant homotopy at $i \circ \Sigma^{n} \theta_{n}^{X}$.

4 and 5: The usual homotopy from the constant map to $-\left(i \circ \varepsilon_{n}\right)+\left(i \circ \varepsilon_{n}\right)$.

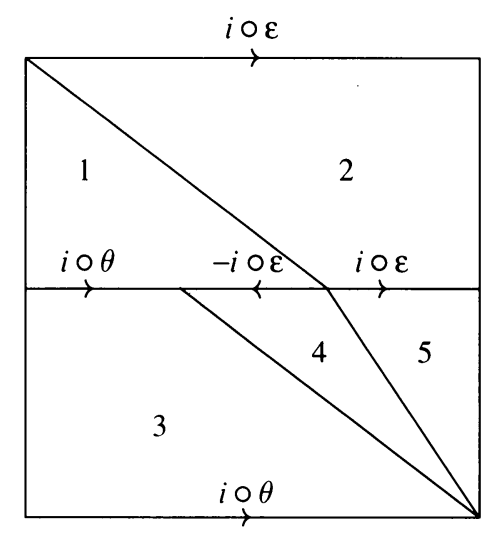

Thus $\Psi_{0}=i \circ \Sigma^{n} \theta_{n}^{X}$ and $\Psi_{1}=i \circ \varepsilon_{n}$. However, $\Psi_{t}(w,[1, x]) \neq i(w, x)$ in general, indeed the picture representing $\Psi: \Sigma^{n} X \wedge I_{+} \rightarrow P_{p}^{n} X$ is

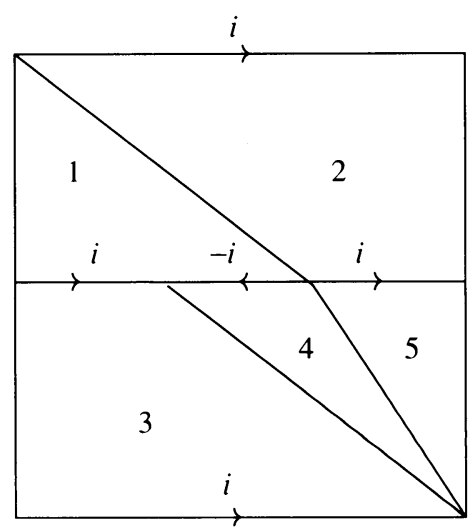

Thus $\Psi$ satisfies the first two properties (4.27), but not the third. However, 
for all, $t$, the following diagram does commute up to homotopy:

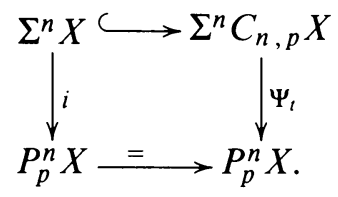

It is not difficult to see that one can construct a natural homotopy $W_{t}: \Sigma^{n} X \wedge$ $I_{+} \rightarrow P_{p}^{n} X$ which makes this diagram commute.

Define $W: \Sigma^{n} X \wedge I_{+} \wedge I_{+} \rightarrow P_{p}^{n} X$ by doing the following things. In the top half $\left(t \geq \frac{1}{2}\right)$ of the square above, the map in region 1 can be coned off within $\Sigma^{n} X \wedge I \wedge I_{+} \subset \Sigma^{n} \widetilde{C}_{n, p} X \wedge I_{+}$(using $s$ as the cone parameter), and region 2 can be stretched to occupy the entire upper half of the square. At the $t=\frac{1}{2}$ line (on the border of region 1), the coning off overlaps with the null-homotopy $i \circ h$ of $i-i$. Here the parameter $u$ must be adjusted so that the 2 vertices on the line $t=\frac{1}{2}$ slide to the left as $s$ increases, shrinking region 1. In the lower half of the square, region 4 shrinks by traversing backwards along the suspension parameter $u$ less and less as $s$ increases. In regioin 3 , the suspension parameter is traversed less as $s$ and $t$ increase, so that when $s=1$ and $t=\frac{1}{2}$ it is not traversed at all. This corresponds to shrinking region 3 and expanding region 5 as $s$ increases. At intermediate values of $s$, for a fixed $t<\frac{1}{2}$, the resulting horizontal slice consists of traversing forward part of the way along the suspension parameter (region 3 ), then going backward for a short time (region 4), and once again going forward to the end (region 5). The speeds are adjusted so that at the end $(s=1)$ the suspension parameter is traversed exactly once in the bottom half of the square (through regions 3 and 5). At an intermediate step (close to $s=1$ ) the homotopy $W$ is represented by the follo:ving picture:

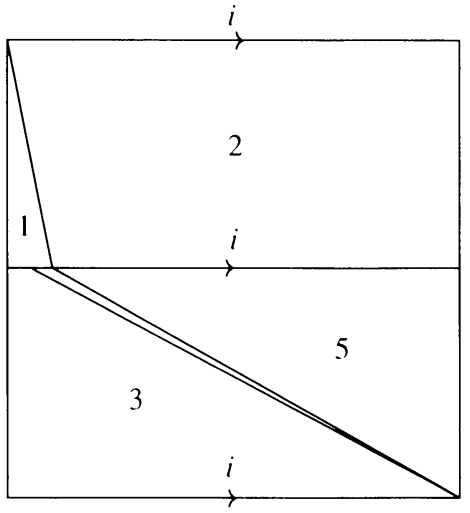

For each point $(u, v, x) \in S^{1} \wedge S^{n-1} \wedge X, W_{s, t}$ satisfies the properties

$$
\begin{gathered}
W_{0, t}(u, v, x)=\Psi_{t}(u, v,[1, x]) ; \quad W_{1, t}(u, v, x)=i(u, v, x) ; \\
W_{s, 0}(u, v, x)=i(u, v, x) ; \quad W_{s, 1}(u, v, x)=i(u, v, x) .
\end{gathered}
$$

If we can show $\left(\Sigma^{n} C_{n, p} X, \Sigma^{n} X\right)$ is an NDR-pair [St], then we can use the homotopy extension property of cofibrations, along with the homotopies $\Psi$ and $W$, to obtain $\widehat{\Phi}$ which satisfies the required properties. Now $\left(\Sigma^{n} C_{n, p} X\right.$, 
$\left.\Sigma^{n} C_{n, 1} X\right)$ is an NDR-pair by [Mas, Proposition 2.6]; therefore it suffices [Ma, Lemma A.5] to show $\left(\Sigma^{n} C_{n, 1} X, \Sigma^{n} X\right)$ is an NDR-pair. A retractile neighborhood of $X$ in $C_{n, 1} X$ is obtained by taking all points $[c, x] \in C_{n, 1} X$ for which the $n$-cube $c$ has a sufficiently large diameter (where diameter means the minimum of the lengths of the edges of $c$ ).

Define a homotopy

$$
\bar{W}: \Sigma^{n} X \wedge I_{+} \wedge I_{+} \cup \Sigma^{n} C_{n, p} X \wedge\{0\} \wedge I_{+} \cup \Sigma^{n} C_{n, p} X \wedge I_{+} \wedge \partial I_{+} \rightarrow P_{p}^{n} X
$$

to be $W$ on $\Sigma^{n} X \wedge I_{+} \wedge I_{+}, \Psi$ on $\Sigma^{n} C_{n, p} X \wedge\{0\} \wedge I_{+}$, the constant homotopy at $i \circ \Sigma^{n} \theta_{n}^{X}$ on $\Sigma^{n} C_{n, p} X \wedge I_{+} \wedge\{0\}$, and the constant homotopy at $i \circ \varepsilon_{n}$ on $\Sigma^{n} C_{n, p} X \wedge I_{+} \wedge\{1\}$. This homotopy has an extension (which we also denote by $\bar{W})$ to all of $\Sigma^{n} C_{n, p} X \wedge I_{+} \wedge I_{+}$, and the restriction of $\bar{W}$ to $\Sigma^{n} C_{n, p} X \wedge\{1\} \wedge I_{+}$ satisfies the properties required for $\widehat{\Phi}$. Q.E.D.

We can now make the following definition.

Definition 4.5. Let $\left(X, \theta_{n}^{X}\right) \in \mathrm{Ob} \mathscr{H}_{p}^{n}$. The $\mathscr{H}_{p}^{n}$ morphism

$$
\eta: X \rightarrow \Omega^{n} P_{p}^{n} X
$$

is defined as the class $[\eta, \Phi]$, where $\eta$ is adjoint to $i$, and $\Phi$ is adjoint to the homotopy $\widehat{\Phi}$ from Lemma 4.4 .

In order for $\eta$ to be well defined, we must check that any choice of the representation of $\left(\Sigma^{n} C_{n, p} X, \Sigma^{n} X\right)$ as an NDR-pair yields the same morphism in $\mathscr{H}_{p}^{n}$. In other words, since $\widehat{\Phi}: \Sigma^{n} C_{n, p} X \wedge I_{+} \rightarrow P_{p}^{n} X$ was defined via the homotopies $\Psi$ and $W$ using the homotopy extension property, we must check that a different choice of the extension $\bar{W}$ yields the same morphism. Suppose $\widehat{\Phi^{\prime}}$ is obtained via a different extension $\bar{W}^{\prime}$ of $W$. Then $\widehat{\Phi}$ and $\widehat{\Phi}^{\prime}$ fit into the pictures in (4.36), which represent $\bar{W}$ and $\bar{W}^{\prime}$ as maps $\Sigma^{n} C_{n, p} X \wedge I_{+} \wedge I_{+} \rightarrow$ $P_{p}^{n} X$.
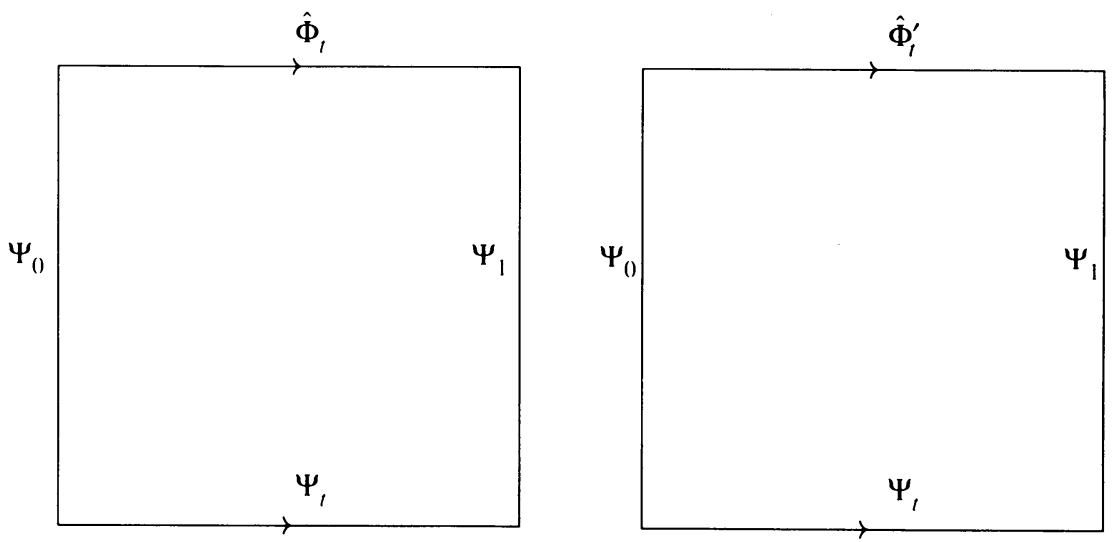

Here the horizontal direction is $t$ and the vertical direction is $s$. Both squares restricted to $\Sigma^{n} X \wedge I_{+} \wedge I_{+}$give the homotopy $W$. We can form a cube from these two squares by connecting the faces corresponding to $\Psi_{0}, \Psi_{1}$, and $\Psi_{t}$ by constant homotopies, and letting the cube restricted to $\Sigma^{n} X \wedge I_{+} \wedge I_{+} \wedge I_{+}$ represent the constant homotopy at $W$. Then we can again use the homotopy 
extension property to fill in the cube. In particular, the top face will yield an equivalence of the $H_{p}^{n}$-functions $(\eta, \Phi)$ and $\left(\eta, \Phi^{\prime}\right)$ (i.e. satisfying (2.10) and (2.11), where we take $P$ to be the constant homotopy at $\eta)$ as desired. Thus $\eta$ is indeed well defined.

Theorem 4.6. Let $\eta: 1 \rightarrow \Omega^{n} P_{p}^{n}$ be the map from Definition 4.5. Then $\eta$ is a natural transformation of functors.

Proof. Let $\left(X, \theta_{n}^{X}\right),\left(Y, \theta_{n}^{Y}\right) \in \mathrm{Ob} \mathscr{H}_{p}^{n}$ and let $[f, F]$ be a morphism from $X$ to $Y$. Then we must show that the following diagram is commutative in the category $\mathscr{H}_{p}^{n}$ :

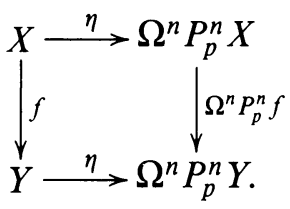

The diagram is strictly commutative as a diagram of spaces; this is easy to see by taking the adjoint diagram

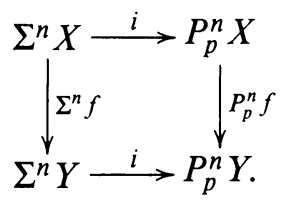

Thus the real content of the theorem is in showing that $\eta \circ f$ and $\Omega^{n} P_{p}^{n} f \circ \eta$ are equivalent as $H_{p}^{n}$-functions.

It is simpler to consider the adjoint situation. Let $\widehat{P}: \Sigma^{n} X \wedge I_{+} \rightarrow P_{p}^{n} Y$ be the constant homotopy which is equal to $i \circ \Sigma^{n} f=P_{p}^{n} f \circ i$. Now in view of (2.10) and (2.11) it suffices to find a homotopy

$$
\widehat{Q}: \Sigma^{n} C_{n, p} X \wedge I_{+} \wedge I_{+} \rightarrow P_{p}^{n} Y
$$

which satisfy the properties

$$
\begin{gathered}
\widehat{Q}_{s, 0}= \begin{cases}i \circ \Sigma^{n} F_{2 s} & \text { if } s \in\left[0, \frac{1}{2}\right] ; \\
\widehat{\Phi}_{2 s-1} \circ \Sigma^{n} C_{n, p} f & \text { if } s \in\left[\frac{1}{2}, 1\right] ;\end{cases} \\
\widehat{Q}_{s, 1}= \begin{cases}P_{p}^{n} f \circ \widehat{\Phi}_{2 s} & \text { if } s \in\left[0, \frac{1}{2}\right] ; \\
P_{p}^{n} f \circ i \circ \varepsilon_{n} & \text { if } s \in\left[\frac{1}{2}, 1\right] ;\end{cases} \\
\widehat{Q}_{0, t}=i \circ \Sigma^{n}\left(f \circ \theta_{n}^{X}\right), \quad \widehat{Q}_{1, t}=i \circ \Sigma^{n} f \circ \varepsilon_{n}, \\
\widehat{Q}_{s, t}(w,[1, x])=i \circ \Sigma^{n} f(w, x) \quad \forall w \in S^{n}, x \in X .
\end{gathered}
$$

In order to produce such a $\widehat{Q}$, we first produce a homotopy

$$
\widehat{Q}^{\prime}: \Sigma^{n} C_{n, p} X \wedge I_{+} \wedge I_{+} \rightarrow P_{p}^{n} Y
$$


which satisfy the properties

$$
\begin{gathered}
\widehat{Q}_{s, 0}^{\prime}= \begin{cases}i \circ \Sigma^{n} F_{2 s} & \text { if } s \in\left[0, \frac{1}{2}\right] ; \\
\Psi_{2 s-1} \circ \Sigma^{n} C_{n, p} f & \text { if } s \in\left[\frac{1}{2}, 1\right] ;\end{cases} \\
\widehat{Q}_{s, 1}^{\prime}= \begin{cases}P_{p}^{n} f \circ \Psi_{2 s} & \text { if } s \in\left[0, \frac{1}{2}\right] ; \\
P_{p}^{n} f \circ i \circ \varepsilon_{n} & \text { if } s \in\left[\frac{1}{2}, 1\right] ;\end{cases} \\
\widehat{Q}_{0, t}^{\prime}=i \circ \Sigma^{n}\left(f \circ \theta_{n}^{X}\right), \quad \widehat{Q}_{1, t}^{\prime}=i \circ \Sigma^{n} f \circ \varepsilon_{n} .
\end{gathered}
$$

We can then use $\widehat{Q}^{\prime}$ to produce a suitable $\widehat{Q}$ via a homotopy extension argument similar to the one used in the proof of Lemma 4.4.

Now $\widehat{Q}_{s, 0}^{\prime}$ can be represented by the picture in (4.43). If the vertical direction is $s$ and the horizontal direction is $u$, then the labelled regions on the picture correspond to the following maps.

1-5: The homotopy $\Psi \circ C_{n, p} f$, where $\Psi$ is the homotopy from the proof of Lemma 4.4 (note $i \circ \varepsilon_{n} \circ \Sigma^{n} C_{n, p} f=i \circ \Sigma^{n} f \circ \varepsilon_{n}$ ).

6: The homotopy $i \circ \Sigma^{n} F$ from $i \circ \Sigma^{n}\left(f \circ \theta_{n}^{X}\right)$ to $i \circ \Sigma^{n}\left(\theta_{n}^{Y} \circ C_{n, p} f\right)$.

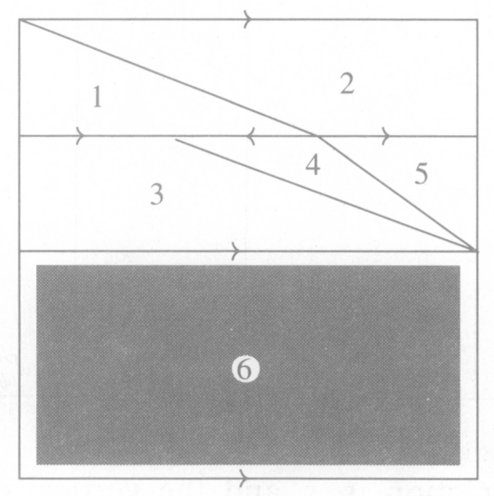

On the other hand, $\widehat{Q}_{s, 1}^{\prime}$ can be represented by the picture in (4.44), where again the labelled regions represent the following maps.

1 and 5: The constant homotopy at $i \circ \varepsilon_{n} \circ \Sigma^{n} C_{n, p} f=i \circ \Sigma^{n} f \circ \varepsilon_{n}$.

2: Coning off of $i \circ\left(\Sigma^{n} \theta_{n}^{Y}-\varepsilon_{n}\right) \circ \Sigma^{n} C_{n, p} f$ within $\Sigma^{n} \widetilde{C}_{n, p} Y \wedge I$.

3: The homotopy $i \circ \Sigma^{n} F$ from $i \circ \Sigma^{n}\left(f \circ \theta_{n}^{X}\right)$ to $i \circ \Sigma^{n}\left(\theta_{n}^{Y} \circ C_{n, p} f\right)$.

4: The constant homotopy at $-\left(i \circ \varepsilon_{n} \circ \Sigma^{n} C_{n, p} f\right)=-\left(i \circ \Sigma^{n} f \circ \varepsilon_{n}\right)$.

6: The constant homotopy at $i \circ \Sigma^{n}\left(f \circ \theta_{n}^{X}\right)$.

7 and 8: The usual homotopy from the constant map to $-\left(i \circ \Sigma^{n} f \circ \varepsilon_{n}\right)+$ $\left(i \circ \Sigma^{n} f \circ \varepsilon_{n}\right)$. 


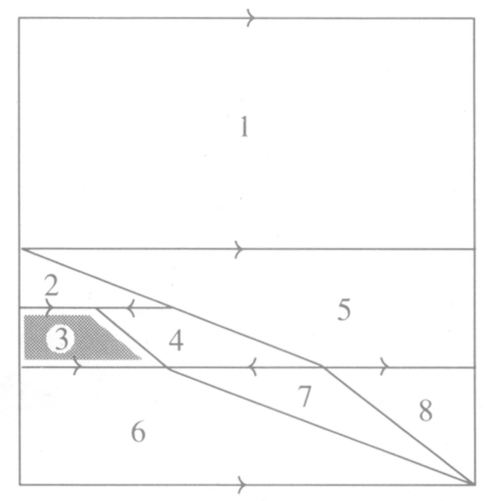

The shaded region in the two pictures corresponds to the homotopy $i \circ \Sigma^{n} F$.

Define the homotopy $\widehat{Q}_{s, t}^{\prime}$ by sliding the shaded region upward and the region corresponding to $\Psi$ downward simultaneously. This can be done without disturbing either the top or bottom of the pictures.

The homotopy $\bar{W}$ from the proof of Lemma 4.4 can be used to construct maps $\Sigma^{n} C_{n, p} X \wedge I_{+} \wedge I_{+} \rightarrow P_{p}^{n} Y$ which are represented by the pictures
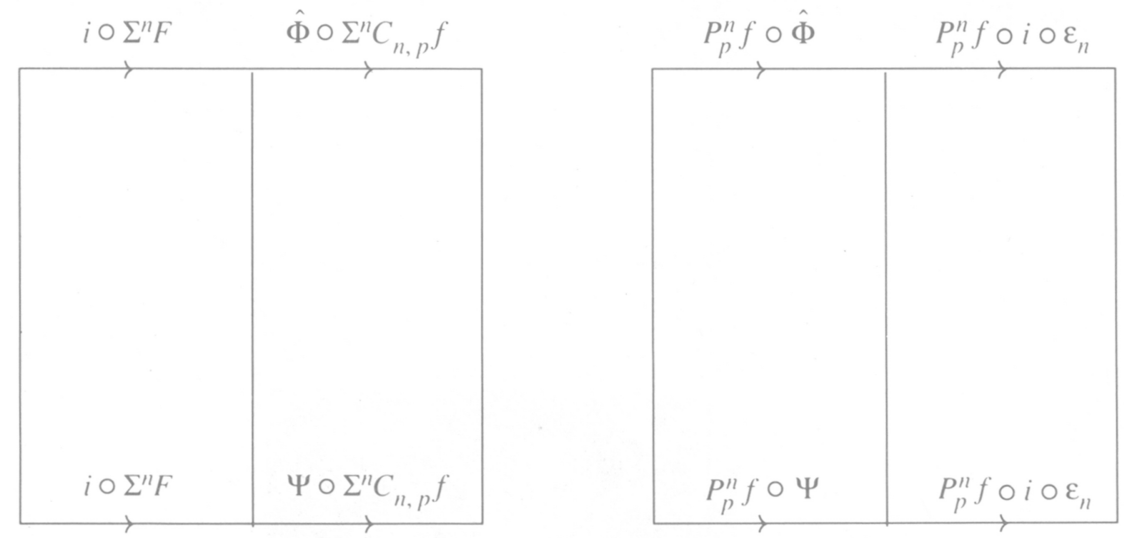

Here the horizontal direction is $s$ and the vertical direction is $r$. The left half of the left square and the right half of the right square are just constant homotopies; and the right half of the left square and the left half of the right square come from $\bar{W}_{r, s}$. The bottom of these two squares $(r=0)$ can be connected by the homotopy $\widehat{Q}_{s, t}^{\prime}$, the left sides $(s=0)$ by a constant homotopy at $i \circ \Sigma^{n}\left(f \circ \theta_{n}^{X}\right)$, and the right sides $(s=1)$ by a constant homotopy at $i \circ \Sigma^{n} f \circ \varepsilon_{n}$. By inspection, the interior of this cube restricted to $\Sigma^{n} X \wedge I_{+} \wedge I_{+} \wedge I_{+}$ can be filled in; i.e. the homotopy $\widehat{Q}^{\prime}$ is compatible with $W$ from the proof of Lemma 4.4. Furthermore, the cube can be filled in in such a way that the top face $(r=1)$ is constant at the map $i \circ \Sigma^{n} f: \Sigma^{n} X \rightarrow P_{p}^{n} Y$. By the homotopy extension property, we can extend the map on the cube to $\Sigma^{n} C_{n, p} X \wedge I_{+} \wedge I_{+} \wedge I_{+}$, and the restriction to the top face $(r=1)$ gives the desired map $\widehat{Q}_{s, t}$. Thus there is a pair $(\widehat{P}, \widehat{Q})$ whose adjoint $(P, Q)$ makes $\eta \circ f$ and $\Omega^{n} P_{p}^{n} f \circ \eta$ equivalent as $H_{p}^{n}$-functions. Q.E.D. 
Theorem 4.7. Let $\left(X, \theta_{n}^{X}\right) \in \mathrm{Ob} \mathscr{H}_{p}^{n}$. Then the composite

$$
P_{p}^{n} X \stackrel{P_{p}^{n} \eta}{\longrightarrow} P_{p}^{n} \Omega^{n} P_{p}^{n} X \stackrel{\epsilon P_{p}^{n}}{\longrightarrow} P_{p}^{n} X
$$

is equal to the identity morphism in $\mathscr{H}_{p}^{0}$.

Proof. On $\Sigma^{n} X$ the composite $\epsilon P_{p}^{n} \circ P_{p}^{n} \eta$ is equal to the composite

$$
\Sigma^{n} X \stackrel{\Sigma^{n} \eta}{\longrightarrow} \Sigma^{n} \Omega^{n} \Sigma^{n} X \stackrel{\epsilon}{\longrightarrow} \Sigma^{n} X
$$

which is (strictly) equal to the identity map.

On $\Sigma^{n} \widetilde{C}_{n, p} X \wedge I$, one can trace through the definitions to see that the composite $\epsilon P_{p}^{n} \circ P_{p}^{n} \eta$ corresponds to the picture in (4.48), where the labelled regions on the picture correspond to the following maps.

1 and 2: The usual homotopy from $\left(i \circ \varepsilon_{n}\right)-\left(i \circ \varepsilon_{n}\right)$ to the constant map.

3-7: The homotopy $\widehat{\Phi}$ of Lemma 4.4 from $i \circ \Sigma^{n} \theta_{n}^{X}$ to $i \circ \varepsilon_{n}$.

8: The constant homotopy at $-\left(i \circ \varepsilon_{n}\right)$.

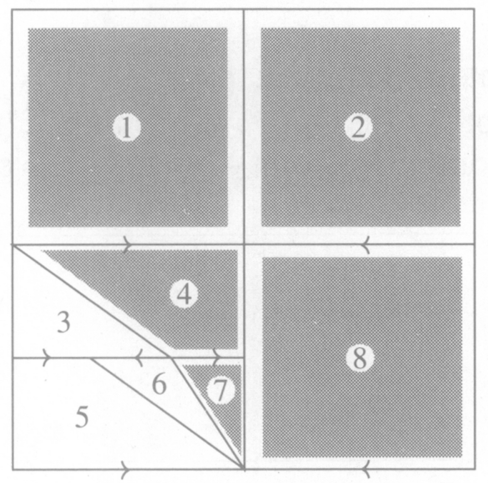

Now this map is homotopic to $P_{p}^{n}$ applied to the identity map of $X$. The required homotopy is defined by shrinking the shaded region of the diagram, so that the right-hand edge of regions 3 and 6 goes to the line $u=1$ and the left-hand edge of region 6 goes to the line $u=\frac{1}{2}$ (and $t \in\left[0, \frac{1}{2}\right]$ ). The end of the homotopy has region 3 replacing the spot formerly held by 1 and 2, 5 replaces 3-7, and 6 replaces 8 . Also, the suspension parameter $u$ in region 6 must be adjusted so that at the end it is traversed completely for all $t \in\left[0, \frac{1}{2}\right]$ (whereas in the beginning it is only traversed part of the way when $t<\frac{1}{4}$ ). Strictly speaking, we should first construct this homotopy with $\widehat{\Phi}$ replaced by $\Psi$ from the proof of Lemma 4.4. Then by being careful about how the shrinking of the shaded region is done (especially regions 4 and 7), we can construct this homotopy to be compatible with the homotopy $W$ from Lemma 4.4. And by using a homotopy extension argument as in the proof of Theorem 4.6, we can obtain a homotopy which has the property that, restricted to $\Sigma^{n} X \wedge I \wedge I$, it stays constant at our usual null-homotopy $h$ of $\operatorname{id}_{\Sigma^{n} X}-\mathrm{id}_{\Sigma^{n} X}$ in the region $t \in\left[0, \frac{1}{2}\right]$. Thus $\epsilon P_{p}^{n} \circ P_{p}^{n} \eta$ is homotopic to $P_{p}^{n}$ applied to the identity map as desired. Q.E.D. 
Theorem 4.8. Let $X \in \mathrm{Ob} \mathscr{H}_{p}^{0}$. Then the composite

$$
\Omega^{n} X \stackrel{\eta \Omega^{n}}{\longrightarrow} \Omega^{n} P_{p}^{n} \Omega^{n} X \stackrel{\Omega^{n} \epsilon}{\longrightarrow} \Omega^{n} X
$$

is equal to the identity morphism in $\mathscr{H}_{p}^{n}$.

Proof. Consider the adjoint of $\Omega^{n} \epsilon \circ \eta \Omega^{n}$, which is the composite

$$
\Sigma^{n} \Omega^{n} X \stackrel{i \Omega^{n}}{\longrightarrow} P_{p}^{n} \Omega^{n} X \stackrel{\epsilon}{\longrightarrow} X .
$$

This composite is equal to $\epsilon: \Sigma^{n} \Omega^{n} X \rightarrow X$ by diagram (4.4) (when $k=0$ ). Therefore the composite $\Omega^{n} \epsilon \circ \eta \Omega^{n}$ is equal to the identity map. Of course this does not necessarily mean that $\Omega^{n} \epsilon \circ \eta \Omega^{n}$ represents the identity morphism in $\mathscr{H}_{p}^{n}$. We must study $\Omega^{n} \epsilon \circ \eta \Omega^{n}$ as an $H_{p}^{n}$-function.

The structure of $\Omega^{n} \epsilon \circ \eta \Omega^{n}$ as an $H_{p}^{n}$-function is determined by the map $\Gamma: C_{n, p} \Omega^{n} X \wedge I_{+} \rightarrow \Omega^{n} X$, where $\Gamma$ is determined by the structures of $\Omega^{n} \epsilon$ and $\eta \Omega^{n}$ as $H_{p}^{n}$-functions. For convenience, we consider the adjoint $\widehat{\Gamma}: \Sigma^{n} C_{n, p} \Omega^{n} X$ $\wedge I_{+} \rightarrow X$. Definitions 3.2 and 4.5, together with the definition (2.9) of the composite of $H_{p}^{n}$-functions tell us that the homotopy $\widehat{\Gamma}$ corresponds to the picture in (4.51), where the labelled regions on the picture correspond to the following maps.

1, 3, and 4: The constant homotopy at $\epsilon \circ \varepsilon_{n}$.

2: Coning off of $\left(\epsilon \circ \varepsilon_{n}\right)-\left(\epsilon \circ \varepsilon_{n}\right)$ within $\Sigma^{n} \widetilde{C}_{n, p} \Omega^{n} X \wedge I$.

5 and 6: The usual homotopy from $-\left(\epsilon \circ \varepsilon_{n}\right)+\left(\epsilon \circ \varepsilon_{n}\right)$ to the constant map.

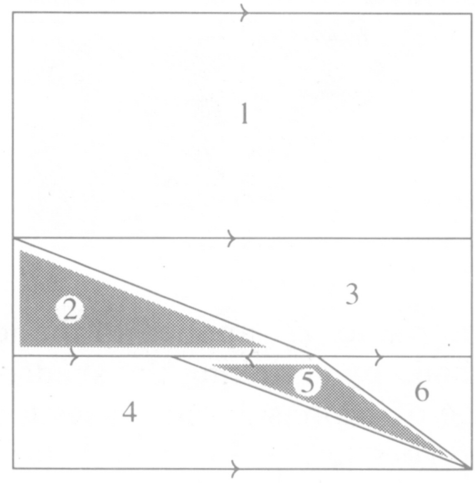

Now it is not difficult to see that $\widehat{\Gamma}$ is homotopic to a map which is the constant homotopy at $\epsilon \circ \varepsilon_{n}$. The required homotopy is defined by shrinking the shaded regions, exactly as in the construction of the homotopy $W$ of Lemma 4.4. This homotopy has the property that it leaves the top and bottom lines above fixed throughout. Again, in order to really construct this homotopy properly, we must consider the picture in (4.51) as belonging to the version of $\eta$ that arises via $\Psi$, rather than $\widehat{\Phi}$. Since this homotopy is compatible with $W$ in the region where $t \in\left[0, \frac{1}{2}\right]$, we can get the actual homotopy that we want by using a homotopy extension argument as in the proofs of Theorems 4.6 and 4.7. This shows that $\Omega^{n} \epsilon \circ \eta \Omega^{n}$ does indeed represent the identity morphism in $\mathscr{H}_{p}^{n}$, since the structure of the identity on $\Omega^{n} X$ as an $H_{p}^{n}$-function is given by the adjoint of the constant homotopy at $\epsilon \circ \varepsilon_{n}$. Q.E.D. 
Theorem 4.9. The functor $P_{p}^{n}: \mathscr{H}_{p}^{n} \rightarrow \mathscr{H}_{p}^{0}$ is left adjoint to $\Omega^{n}: \mathscr{H}_{p}^{0} \rightarrow \mathscr{H}_{p}^{n}$. Proof. This follows from Theorems 4.3, 4.6, 4.7, 4.8, and standard category theory. Q.E.D.

\section{THE CASE $n=\infty$}

In this section we briefly discuss how the results in $\S \S 3$ and 4 extend to the case in which $n=\infty$.

Let $\mathscr{H} \mathscr{S}$ denote the homotopy category of spectra. Then it is well known that $\Omega^{\infty}$ is a functor $\mathscr{H} \mathscr{S} \rightarrow \mathscr{H}_{p}^{\infty}$ (on objects $\Omega^{\infty}$ is the zero space of the spectrum). The precise definition of $\Omega^{\infty}$ is essentially the same as the definition of $\Omega^{n}$ when $n$ is finite. The left adjoint of $\Omega^{\infty}$ will be a functor $\mathbf{P}_{\mathbf{p}}: \mathscr{H}_{p}^{\infty} \rightarrow \mathscr{H} \mathscr{S}$.

In order to define $\mathbf{P}_{\mathbf{p}}$ we use Theorem 4.9 in the following way. Suppose $\left(X, \theta_{\infty}^{X}\right) \in \mathrm{Ob} \mathscr{H}_{p}^{\infty}$. Then it is easy to see that it is also the case that $\left(X, \theta_{n}^{X}\right) \in \mathrm{Ob} \mathscr{H}_{p}^{n}$ for every finite $n$, where $\theta_{n}^{X}$ is the restriction of $\theta_{\infty}^{X}$ to $C_{n, p} X$. Thus for every finite $n, \eta: X \rightarrow \Omega^{n+1} P_{p}^{n+1} X$ is an $H_{p}^{n}$-map which, by Theorem 4.9, is adjoint to a map $P_{p}^{n} X \rightarrow \Omega P_{p}^{n+1} X$. We can then define $\mathbf{P}_{\mathbf{p}} X$ to be the spectrum whose $k$ th-space is $\underset{n}{\lim } \Omega^{n} P_{p}^{n+k} X$. The definition of $\mathbf{P}_{\mathbf{p}}$ on morphisms is the obvious one. It is now completely formal that the analogues of all of the results of $\S 4$ hold.

To relate this definition of $\mathbf{P}_{\mathrm{p}} X$ to our basic cofibration sequence, we have the analogue of Proposition 3.7.

Proposition 5.1. For $\left(X, \theta_{\infty}^{X}\right) \in \mathrm{Ob} \mathscr{H}_{p}^{\infty}$, let $h: \Sigma^{\infty} \widetilde{C}_{\infty, p} X \rightarrow \Sigma^{\infty} X$ be the unique map making the diagram

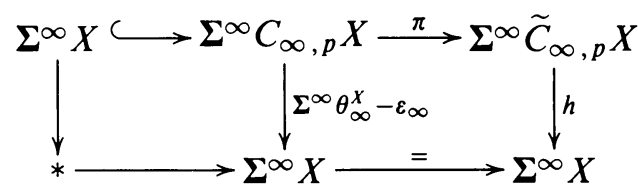

homotopy commute.Then there is a natural cofibration sequence

$$
\boldsymbol{\Sigma}^{\infty} \tilde{C}_{\infty, p} X \stackrel{h}{\longrightarrow} \boldsymbol{\Sigma}^{\infty} X \stackrel{i}{\rightarrow} \mathbf{P}_{\mathbf{p}} X \stackrel{j}{\longrightarrow} \boldsymbol{\Sigma} \boldsymbol{\Sigma}^{\infty} \tilde{C}_{\infty, p} X .
$$

From this we can conclude that $h$ can be interpreted as a "generalized KahnPriddy map" as follows. Let $D_{p} X=C_{\infty, p} X / C_{\infty, p-1} X$. Given an $H_{p}^{\infty}$-space $X$ and $q \leq p$, define a map $f_{q}: \Sigma^{\infty} D_{q} X \rightarrow \Sigma^{\infty} X$ as the composite $\Sigma^{\infty} D_{q} X \hookrightarrow$ $\Sigma^{\infty} C_{\infty, p} X \stackrel{\Sigma^{\infty} \theta_{\infty}^{X}}{\longrightarrow} \Sigma^{\infty} X$, where the first map arises from the Kahn-Snaith splitting [CMT]

$$
\boldsymbol{\Sigma}^{\infty} C_{\infty, p} X \simeq \bigvee_{1 \leq q \leq p} \boldsymbol{\Sigma}^{\infty} D_{q} X
$$

Corollary 5.2. For $\left(X, \theta_{\infty}^{X}\right) \in \mathrm{Ob} \mathscr{K}_{p}^{\infty}$, there is a homotopy commutative diagram 


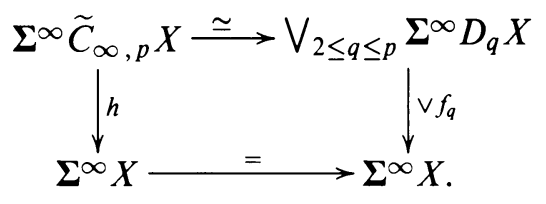

When $X=\Omega^{\infty} \mathbf{E}$, the maps $f_{q}$ were studied in [Ku2]. In particular, when $X=S^{0}, f_{2}: \Sigma^{\infty} D_{2} S^{0} \rightarrow \Sigma^{\infty} S^{0}$ can be identified with the Kahn-Priddy map $\Sigma^{\infty} R P_{+}^{\infty} \rightarrow \Sigma^{\infty} S^{0}$, so that $\mathbf{P}_{2} S^{0}$ will be $\Sigma \mathbf{P}_{-1}^{\infty}$ [Mh] (see Example 7.6).

We note for the next section that the discussion above gives an obvious map

$$
\Sigma^{-n} \boldsymbol{\Sigma}^{\infty} P_{p}^{n} X \rightarrow \mathbf{P}_{\mathbf{p}} X
$$

and it is not difficult to see that $\mathbf{P}_{\mathbf{p}} X=\underset{n}{\underset{n}{\lim }} \Sigma^{-n} \Sigma^{\infty} P_{p}^{n} X$. This gives us a natural filtration of $\mathbf{P}_{\mathbf{p}} X$,

$$
\Sigma^{\infty} X \rightarrow \Sigma^{-1} \Sigma^{\infty} P_{p}^{1} X \rightarrow \cdots \rightarrow \Sigma^{-n} \Sigma^{\infty} P_{p}^{n} X \rightarrow \cdots \rightarrow \mathbf{P}_{\mathbf{p}} X,
$$

which is used to help interpret the calculation of $\widetilde{H}^{*}\left(\mathbf{P}_{2} X ; \mathbf{F}_{2}\right)$. This is the same filtration that arises via the cofibration sequences (3.18) from the filtration

$$
X \rightarrow C_{1, p} X \rightarrow \cdots \rightarrow C_{n, p} X \rightarrow \cdots \rightarrow C_{\infty, p} X,
$$

where the maps above (except for the first one) arise via the inclusions $I \hookrightarrow$ $I^{2} \hookrightarrow I^{3} \cdots$. Thus we deduce that, for $0 \leq k<n \leq \infty$, there are homotopy equivalences of cofibers

$$
P_{p}^{n} X / \Sigma^{n-k} P_{p}^{k} X \simeq \Sigma^{n+1} \widetilde{C}_{n, p} X / \widetilde{C}_{k, p} X
$$

When $p=2$, this last cofiber was identified in [Kul, Proposition 1.3.2].

Proposition 5.3. The sequence

$$
\Sigma^{k} \widetilde{C}_{k, 2} X \rightarrow \Sigma^{k} \widetilde{C}_{n, 2} X \stackrel{\varepsilon_{k}}{\longrightarrow} \widetilde{C}_{n-k, 2} \Sigma^{k} X
$$

is homotopic to a cofibration sequence.

\section{6. Сономоlogy}

In this section we use the results of the previous sections to deduce information about $E^{*}(X)$ and $E^{*}\left(P_{p}^{n} X\right)$, where $E^{*}$ is a generalized cohomology theory and $X \in \mathrm{Ob} \mathscr{H}_{p}{ }^{n}$. Our strongest results come when we specialize to the case $E^{*}(X)=\widetilde{H}^{*}\left(X ; \mathbf{F}_{2}\right)$. In this case, $\widetilde{H}_{*}\left(X ; \mathbf{F}_{2}\right)$ admits Dyer-Lashof operations, and, roughly put, we show that trivial Dyer-Lashof operations in $\widetilde{H}_{*}\left(X, \mathbf{F}_{2}\right)$ imply nontrivial Steenrod operations in $\widetilde{H}^{*}\left(P_{2}^{n} X ; \mathbf{F}_{2}\right)$ (and vice versa).

Let $E_{q}$ be the space representing $E^{q}(X)$, i.e. $\left[X, E_{q}\right]=E^{q}(X)$, and let $\sigma^{n}: E^{q}(X) \rightarrow E^{q+n}\left(\Sigma^{n} X\right)$ be the suspension isomorphism.

We first show that our adjunction results allow us to analyze when a map $f: X \rightarrow E_{q}$, representing an element $x \in E^{q}(X)$, admits the structure of an $H_{p}^{n}$-map (when $X \in \mathrm{Ob} \mathscr{H}_{p}^{n}$ ). Indeed, if $X$ has fundamental cofibration sequence

$$
\Sigma^{n} \widetilde{C}_{n, p} X \stackrel{h}{\longrightarrow} \Sigma^{n} X \stackrel{i}{\longrightarrow} P_{p}^{n} X \stackrel{j}{\longrightarrow} \Sigma^{n+1} \widetilde{C}_{n, p} X
$$


then Theorem 4.9 shows that we have a natural bijection

$$
\left[X, E_{q}\right]_{\mathscr{P}_{p}^{n}} \cong E^{q+n}\left(P_{p}^{n} X\right) .
$$

Furthermore, the forgetful map

$$
\left[X, E_{q}\right]_{\mathscr{Z}_{p}} \rightarrow\left[X, E_{q}\right],
$$

which sends an $H_{p}^{n}$-map $[f, F]$ to the underlying map $f$, can be identified with the composite

$$
E^{q+n}\left(P_{p}^{n} X\right) \stackrel{i^{*}}{\longrightarrow} E^{q+n}\left(\Sigma^{n} X\right) \stackrel{\left(\sigma^{n}\right)^{-1}}{\longrightarrow} E^{q}(X) .
$$

Thus we have the following theorem.

Theorem 6.1. If $x \in E^{q}(X)$ is represented by $f: X \rightarrow E_{q}$, then $f$ can be given the structure of an $H_{p}^{n}$-map if and only if $h^{*}(x)=0$. Furthermore, the elements of Mor $\mathscr{H}_{p}^{n}$ whose underlying map is $f$ are in 1-1 correspondence with elements $\bar{x} \in E^{q+n}\left(P_{p}^{n} X\right)$ for which $i^{*}(\bar{x})=\sigma^{n} x$.

Similarly, for $k<n$, one can analyze when an $H_{p}^{k}$-structure on a map $f: X \rightarrow E_{q}$, corresponding to an element $x \in E^{q}(X)$, can be extended to an $H_{p}^{n}$-structure. As before, we are asking whether a given lift $\bar{x} \in E^{q+k}\left(P_{p}^{k} X\right)$ lifts further to $E^{q+n}\left(P_{p}^{n} X\right)$ under the map induced by $\Sigma^{n-k} P_{p}^{k} X \rightarrow P_{p}^{n} X$. As observed in (5.8), the cofiber of this map is naturally homotopy equivalent to $\Sigma^{n+1} \widetilde{C}_{n, p} X / \widetilde{C}_{k, p} X$. Thus we have the following theorem.

Theorem 6.2. If $X \in \mathrm{Ob} \mathscr{H}_{p}^{n}$ and $x \in E^{q}(X)$ is represented by $f: X \rightarrow E_{q}$, then the obstruction to a given $H_{p}^{k}$-structure on $f$ extending to an $H_{p}^{n}$-structure is an element of $E^{q}\left(\widetilde{C}_{n, p} X / \widetilde{C}_{k, p} X\right)$.

Combined with Proposition 5.3, this theorem has the following corollary.

Corollary 6.3. If $X \in \mathrm{Ob} \mathscr{H}_{2}^{n}$ and $x \in E^{q}(X)$ is represented by $f: X \rightarrow E_{q}$, then the obstruction to a given $H_{2}^{k}$-structure on $f$ extending to an $H_{2}^{n}$-structure is an element of $E^{q+k}\left(\widetilde{C}_{n-k, 2} \Sigma^{k} X\right)$. In particular, the obstruction to a given $H_{2}^{n-1}$-structure extending to an $H_{2}^{n}$-structure is an element of $E^{q+1-n}(X \wedge X)$.

The last statement of Corollary 6.3 is a version of the main theorem of [S11], where this obstruction is called the $h_{n-1}$-deviation, and denoted $h_{n-1}(f)$.

Now we turn our attention to $\widetilde{H}^{*}\left(P_{2}^{n} X ; \mathbf{F}_{2}\right)$. When $n=1$, a theorem of Thomas [Th] (see Appendix B) implies the following result for the usual Hopf construction sequence. Thomas' result can also be used to prove Theorem 6.4, since our map $h$ agrees stably with the Hopf construction, but we given an independent proof here, which uses Theorem 4.9, and will allow for generalization to odd primes.

Theorem 6.4. Suppose $X$ is an $H$-space, with basic cofibration sequence

$$
\Sigma(X \wedge X) \stackrel{h}{\longrightarrow} \Sigma X \stackrel{i}{\rightarrow} P_{2}^{1} X \stackrel{j}{\longrightarrow} \Sigma^{2}(X \wedge X) .
$$

If $\bar{x} \in \widetilde{H}^{q+1}\left(P_{2}^{1} X ; \mathbf{F}_{2}\right)$, then

$$
S q^{q+1} \bar{x}=j^{*}\left(\sigma^{2}(x \otimes x)\right),
$$

where $i^{*} \bar{x}=\sigma x$ defines $x \in \widetilde{H}^{q}\left(X ; \mathbf{F}_{2}\right)$. 
Proof. Note that in the sequence above, we have identified $\Sigma \widetilde{C}_{1,2} X$ with the homotopy equivalent space $\Sigma(X \wedge X)$.

We give the proof for the universal example, $X=K(\mathbf{Z} / 2, q)$ and $x=l_{q}$. Let $\bar{l}_{q} \in \widetilde{H}^{q+1}\left(P_{2}^{1} K(\mathbf{Z} / 2, q) ; \mathbf{F}_{2}\right)$ be the unique class for which $i^{*}\left(\bar{l}_{q}\right)=\sigma l_{q}$. We first show that $S q^{q+1} \bar{l}_{q} \neq 0$. Let $f: P_{2}^{1} K(\mathbf{Z} / 2, q) \rightarrow K(\mathbf{Z} / 2, q+1)$ represent $\bar{l}_{q}$ and let $E$ be the homotopy fiber of the map $K(\mathbf{Z} / 2, q+1)$ $\stackrel{S q^{q+1}}{\longrightarrow} K(\mathbf{Z} / 2,2 q+2)$. If we suppose that $S q^{q+1} \bar{l}_{q}=0$, then there is a lifting $\bar{f}: P_{2}^{1} K(\mathbf{Z} / 2, q) \rightarrow E$ of $f$ whose adjoint (by Theorem 4.9) $K(\mathbf{Z} / 2, q) \rightarrow \Omega E$ makes $K(\mathbf{Z} / 2, q)$ into a retract of $\Omega E$ in the category $\mathscr{H}_{2}{ }^{1}$. Now it is easy to see that $\Omega E \simeq K(\mathbf{Z} / 2, q) \times K(\mathbf{Z} / 2,2 q)$. However, this splitting does not hold in the category $\mathscr{H}_{2}^{1}$. Indeed, in mod 2 homology, the square of the fundamental class of $K(\mathbf{Z} / 2, q)$ is the fundamental class of $K(\mathbf{Z} / 2,2 q)$. Thus $S q^{q+1} \bar{l}_{q} \neq 0$.

The fact that the square of the fundamental class of $K(\mathbf{Z} / 2, q)$ is the fundamental class of $K(\mathbf{Z} / 2,2 q)$ can be seen via the following argument. In $H^{*}\left(E, \mathbf{F}_{\mathbf{2}}\right)$, there is a unique nonzero $q+1$ dimensional class, and the square of this class is zero. Hence, as an algebra,

$$
H^{*}\left(E, \mathbf{F}_{2}\right) \cong \Lambda(x) \otimes A,
$$

where $x$ is the $q+1$ dimensional class, $s x$ has bidegree $(-1, q+1)$, and $A$ is a Hopf algebra. The $E_{2}$ term of the Eilenberg-Moore spectral sequence converging (as a Hopf algebra) to the cohomology of $\Omega E$ is then isomorphic as a coalgebra to

$$
\Gamma(s x) \otimes \operatorname{Tor}_{A}\left(\mathbf{F}_{\mathbf{2}}, \mathbf{F}_{\mathbf{2}}\right),
$$

where $\Gamma($ ) denotes a divided power coalgebra. Now because it lies in (external) filtration degree -2 , the element $\gamma_{2}(s x)$ is a permanent cycle, and

$$
\Delta\left(\gamma_{2}(s x)\right)=1 \otimes \gamma_{2}(s x)+\gamma_{2}(s x) \otimes 1+s x \otimes s x .
$$

Now $s x$ survives to be the fundamental class of $K(\mathbf{Z} / 2, q)$; hence $\gamma_{2}(s x)$ survives since it is not primitive. Because its coproduct contains the term $s x \otimes$ $s x$, the only possibility for $\gamma_{2}(s x)$ is that it becomes the fundamental class of $K(\mathbf{Z} / 2,2 q)$. The desired conclusion then follows from the coproduct formula (6.9).

Now since $i^{*}\left(S q^{q+1} \bar{l}_{q}\right)=S q^{q+1} \sigma l_{q}=0, S q^{q+1} \bar{l}_{q}$ must be in the image of $j^{*}$, and the only nonzero class of $\widetilde{H}^{2 q+2}\left(\Sigma^{2}(K(\mathbf{Z} / 2, q) \wedge K(\mathbf{Z} / 2, q)) ; \mathbf{F}_{2}\right)$ is $\sigma^{2}\left(l_{q} \otimes l_{q}\right)$. Thus the results holds for $K(\mathbf{Z} / 2, q)$.

For the general case, $\sigma x \in \operatorname{im} i^{*}$ implies that $h^{*}(x)=0$, and hence $x$ is representable by an $H_{2}^{1}$-map $g: X \rightarrow K(\mathbf{Z} / 2, q)$ (whose structure as an $H_{2}^{1}$ map corresponds to the chosen class $\bar{x})$ by Theorem 6.1. Then $\left(P_{2}^{1} g\right)^{*}\left(\bar{l}_{q}\right)=\bar{x}$, and hence $\left(P_{2}^{1} g\right)^{*}\left(S q^{q+1} \bar{l}_{q}\right)=S q^{q+1} \bar{x}$. The homotopy commutative diagram

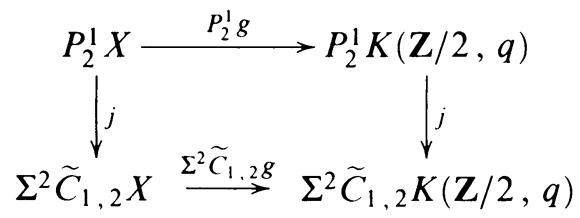

then gives the desired result. Q.E.D. 
We remark that Theorem 6.4 is actually somewhat weaker than Thomas's original result, which gives a similar formula for all cup products, not just the cup product square.

In order to give a generalization of Theorem 6.4 for all $n$, we need some definitions and results concerning mod 2 Dyer-Lashof operations.

Definition 6.5. For every $1 \leq n \leq \infty$ and any space $X$, let

$$
\widetilde{Q}_{r}: \widetilde{H}^{q}\left(X ; \mathbf{F}_{2}\right) \rightarrow \widetilde{H}^{2 q+r}\left(\widetilde{C}_{n, 2} X ; \mathbf{F}_{2}\right)
$$

be defined as follows. If $x \in \widetilde{H}^{q}\left(X ; \mathbf{F}_{2}\right)$ is represented by $f: X \rightarrow K(\mathbf{Z} / 2, q)$, let $\sigma^{r}\left(\widetilde{Q}_{r} x\right)$ be represented by the composite

$$
\begin{aligned}
\Sigma^{r} \widetilde{C}_{n, 2} X \rightarrow \Sigma^{r} \widetilde{C}_{\infty, 2} X \stackrel{\tilde{\varepsilon}_{r}}{\rightarrow} \tilde{C}_{\infty, 2} \Sigma^{r} X \\
\stackrel{\widetilde{C}_{\infty, 2} \Sigma^{r} f}{\longrightarrow} \widetilde{C}_{\infty, 2} \Sigma^{r} K(\mathbf{Z} / 2, q) \rightarrow K(\mathbf{Z} / 2,2 q+2 r),
\end{aligned}
$$

where the last map represents a generator in the lowest dimension for which the graded vector space $\widetilde{H}^{*}\left(\widetilde{C}_{\infty, 2} \Sigma^{r} K(\mathbf{Z} / 2, q) ; \mathbf{F}_{2}\right)$ is nonzero.

The element $\widetilde{Q}_{r} x$ is sometimes denoted $e_{r} \otimes x \otimes x$ or $e_{r} \int x$ in the literature. We remark for future reference that when $r \geq n$ above, the operation $\widetilde{Q}_{r}$ is trivial, since the composite of the first two maps in the definition above will be null-homotopic by Proposition 5.3. One can think of $\widetilde{Q}_{r}$ as a sort of "dual external Dyer-Lashof operation", as we now make more precise.

Recall from [CLM] that $\widetilde{H}_{*}\left(C_{n, 2} X ; \mathbf{F}_{2}\right)$ is additively generated by the image of the map induced by inclusion $\widetilde{H}_{*}\left(X ; \mathbf{F}_{2}\right) \hookrightarrow \widetilde{H}_{*}\left(C_{n, 2} X ; \mathbf{F}_{2}\right)$, together with the external Dyer-Lashof operations

$$
\bar{Q}_{r}: \widetilde{H}_{q}\left(X ; \mathbf{F}_{2}\right) \rightarrow \widetilde{H}_{2 q+r}\left(C_{n, 2} X ; \mathbf{F}_{\mathbf{2}}\right), \quad r \leq n-1,
$$

and iterations of the external Browder and Pontryagin product operations. Both the Browder and Pontryagin product operations are two-variable operations

$$
\widetilde{H}_{*}\left(X ; \mathbf{F}_{2}\right) \otimes \widetilde{H}_{*}\left(X ; \mathbf{F}_{2}\right) \rightarrow \widetilde{H}_{*}\left(C_{n, 2} X ; \mathbf{F}_{2}\right) .
$$

Using the retraction $\varepsilon_{n}: \Sigma^{n} C_{n, 2} X \rightarrow \Sigma^{n} X$, we get a natural decomposition

$$
\widetilde{H}_{*}\left(C_{n, 2} X ; \mathbf{F}_{2}\right) \cong \widetilde{H}_{*}\left(X ; \mathbf{F}_{2}\right) \oplus \widetilde{H}_{*}\left(\widetilde{C}_{n, 2} X ; \mathbf{F}_{2}\right),
$$

where $\widetilde{H}_{*}\left(\widetilde{C}_{n, 2} X ; \mathbf{F}_{2}\right)$ has been identified with $\operatorname{ker}\left(\varepsilon_{n}\right)_{*}$.

Lemma 6.6. For all $v, w \in \widetilde{H}_{*}\left(X ; \mathbf{F}_{2}\right)$, the Dyer-Lashof, Browder, and Pontryagin product operations applied to $v$ and $w$ are all contained in $\operatorname{ker}\left(\varepsilon_{n}\right)_{*}$.

Proof. The result is classical for Pontryagin products, and is closely related to Theorem 4.1 (ii). The diagram

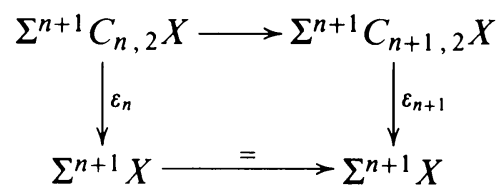


shows that all Browder operations are in $\operatorname{ker}\left(\varepsilon_{n}\right)_{*}$, as they are in the kernel of the induced map $\widetilde{H}_{*}\left(C_{n, 2} X ; \mathbf{F}_{2}\right) \rightarrow \widetilde{H}_{*}\left(C_{n+1,2} X ; \mathbf{F}_{2}\right)$ from above (see [CLM, III.1.2(1)]).

As for the Dyer-Lashof operations, the composite

$$
\widetilde{H}_{q}\left(X ; \mathbf{F}_{\mathbf{2}}\right) \stackrel{\bar{Q}_{r}}{\longrightarrow} \widetilde{H}_{2 q+r}\left(C_{n, 2} X ; \mathbf{F}_{2}\right) \stackrel{\left(\varepsilon_{n}\right) *}{\longrightarrow} \widetilde{H}_{2 q+r}\left(X ; \mathbf{F}_{2}\right)
$$

will be a natural positive-dimensional homology operation, and thus must be identically zero. Q.E.D.

Corollary 6.7. The external Dyer-Lashof, Browder, and Pontryagin operations can be viewed as taking values in $\widetilde{H}_{*}\left(\widetilde{C}_{n, 2} X ; \mathbf{F}_{2}\right)$.

The next proposition relates our external cohomology operations $\widetilde{Q}_{r}$ to the external homology operations, via the Kronecker pairing $\langle$,$\rangle .$

Proposition 6.8. Suppose $x \in \widetilde{H}^{*}\left(X ; \mathbf{F}_{2}\right)$ and $v, w \in \widetilde{H}_{*}\left(X ; \mathbf{F}_{2}\right)$. Then

$$
\left\langle\widetilde{Q}_{r} x, \widetilde{Q}_{s} v\right\rangle= \begin{cases}\langle x, v\rangle & \text { if } r=s \\ 0 & \text { if } r \neq s\end{cases}
$$

Furthermore the element $\widetilde{Q}_{r} x$ paired with any Browder or Pontryagin product operation yields zero, except when $r=0$, in which case

$$
\left\langle\widetilde{Q}_{0} x, v * w\right\rangle=\langle x, v\rangle \cdot\langle x, w\rangle,
$$

where $v * w$ denotes the Pontryagin product.

Proof. When $r>0, \widetilde{Q}_{r} x$ is in the kernel of the map $H^{*}\left(\widetilde{C}_{n, 2} X ; \mathbf{F}_{2}\right) \rightarrow$ $\widetilde{H}^{*}\left(\widetilde{C}_{1,2} X ; \mathbf{F}_{2}\right)$ induced by inclusion. Thus the pairing of $\widetilde{Q}_{r} x$ with any Pontryagin product operation applied to $(v, w)$ must be zero. Similarly, since $\widetilde{Q}_{r} x$ comes from the cohomology of $\widetilde{C}_{\infty, 2} X$, it must pair trivially with any Browder operation.

Using the fact [CLM, (III 1.4)] that the Dyer-Lashof (homology) operations commute appropriately with the evaluation maps $\tilde{\varepsilon}_{k}: \Sigma^{k} \widetilde{C}_{n, 2} X \rightarrow \widetilde{C}_{n-k, 2} \Sigma^{k} X$, the remaining formulas can be reduced to the specific case in which $r=0$, $X=K(\mathbf{Z} / 2, q)$, and $x$ is the fundamental class, where the conclusion is obvious. Q.E.D.

If $\left(X, \theta_{n}^{X}\right) \in \mathrm{Ob} \mathscr{H}_{2}^{n}$, then the structure map $\theta_{n}^{X}$ can be used to define internal Dyer-Lashof operations in $\widetilde{H}_{*}\left(X ; \mathbf{F}_{2}\right)$, for $r \leq n-1$,

$$
Q_{r} v=\left(\theta_{n}^{X}\right)_{*}\left(\bar{Q}_{r} v\right) \text {. }
$$

Recalling the commutative diagram (3.19) from Proposition 3.7, Lemma 6.6 and Proposition 6.8 imply the next result.

Proposition 6.9. $h_{*}\left(\bar{Q}_{r} v\right)=Q_{r} v$. Thus if $\widetilde{Q}_{r} x=h^{*}(y)$ for some $x, y \in$ $\widetilde{H}^{*}\left(X ; \mathbf{F}_{\mathbf{2}}\right)$, then $\langle x, v\rangle=\left\langle y, Q_{r} v\right\rangle$ for all $v \in \widetilde{H}_{*}\left(X ; \mathbf{F}_{\mathbf{2}}\right)$.

We now return to our generalization of Theorem 6.4. When $n=1$ and $x \in \widetilde{H}^{q}\left(X ; \mathbf{F}_{2}\right), \widetilde{Q}_{0}(x)=x \otimes x \in \widetilde{H}^{2 q}\left(\widetilde{C}_{1,2} X ; \mathbf{F}_{2}\right)$. Thus the following theorem generalizes Theorem 6.4. 
Theorem 6.10. Suppose $X \in \mathrm{Ob} \mathscr{H}_{2}^{n}$, with basic cofibration sequence

$$
\Sigma^{n} \widetilde{C}_{n, 2} X \stackrel{h}{\longrightarrow} \Sigma^{n} X \stackrel{i}{\rightarrow} P_{2}^{n} X \stackrel{j}{\longrightarrow} \Sigma^{n+1} \widetilde{C}_{n, 2} X .
$$

If $\bar{x} \in \widetilde{H}^{q+n}\left(P_{2}^{n} X ; \mathbf{F}_{2}\right)$, then

$$
S q^{q+n} \bar{x}=j^{*}\left(\sigma^{n+1}\left(\widetilde{Q}_{n-1} x\right)\right),
$$

where $i^{*} \bar{x}=\sigma^{n} x$ defines $x \in \widetilde{H}^{q}\left(X ; \mathbf{F}_{2}\right)$. In particular, if $S q^{q+n} \bar{x}=0$, then there is an element $y \in \widetilde{H}^{2 q+n-1}\left(X ; \mathbf{F}_{2}\right)$ satisfying $\langle x, v\rangle=\left\langle y, Q_{n-1} v\right\rangle$ for all $v \in \widetilde{H}_{q}\left(X ; \mathbf{F}_{2}\right)$.

Proof. The proof is first given for the universal example $X=K(\mathbf{Z} / 2, q)$ and $x=l_{q}$; the universal example is then used to prove the theorem for general $X$.

The case in which $n=1$ is proved in Theorem 6.4. Consider the case in which $n>1$. Theorem 4.1 implies that the following diagram commutes up to homotopy:

$$
\begin{array}{crr}
P_{2}^{n} K(\mathbf{Z} / 2, q) & j & \Sigma^{n+1} \widetilde{C}_{n, 2} K(\mathbf{Z} / 2, q) \\
\downarrow \varepsilon & \downarrow \Sigma^{2}\left(\widetilde{C}_{1,2} \in \tilde{\varepsilon}_{n-1}\right) \\
P_{2}^{1} K(\mathbf{Z} / 2, q+n-1) & & j \\
\longrightarrow & \Sigma^{2} \widetilde{C}_{1,2} K(\mathbf{Z} / 2, q+n-1) .
\end{array}
$$

Since the choices of $\bar{l}_{q}$ and $\bar{l}_{q+n-1}$ are unique, one sees that $\epsilon^{*}\left(\bar{l}_{q+n-1}\right)=\bar{l}_{q}$, and hence $\epsilon^{*}\left(S q^{q+n} \bar{l}_{q+n-1}\right)=S q^{q+n} \bar{l}_{q}$. By the construction of our external operations,

$$
\left(\Sigma^{2}\left(\widetilde{C}_{1,2} \epsilon \circ \widetilde{\varepsilon}_{n-1}\right)\right)^{*}\left(\sigma^{2}\left(\widetilde{Q}_{0} l_{q+n-1}\right)\right)=\sigma^{n+1}\left(\widetilde{Q}_{n-1} l_{q}\right) ;
$$

thus Theorem 6.4 implies

$$
\begin{aligned}
j^{*}\left(\sigma^{n+1}\left(\tilde{Q}_{n-1} l_{q}\right)\right) & =j^{*} \circ\left(\Sigma^{2}\left(\tilde{C}_{1,2} \epsilon \circ \tilde{\varepsilon}_{n-1}\right)\right)^{*}\left(\sigma^{2}\left(\tilde{Q}_{0 l_{q+n-1}}\right)\right) \\
& =\epsilon^{*} \circ j^{*}\left(\sigma^{2}\left(\widetilde{Q}_{0 l_{q+n-1}}\right)\right) \\
& =\epsilon^{*}\left(S q^{q+n} \bar{l}_{q+n-1}\right)=S q^{q+n} \bar{l}_{q} .
\end{aligned}
$$

This completes the proof for the universal example.

For the general case, Theorem 6.1 implies that $\bar{x}$ corresponds to an $H_{2}^{n}$ structure on a map $f: X \rightarrow K(\mathbf{Z} / 2, q)$ representing $x$ (note that if $i^{*}(\bar{x})=0$, then $\bar{x} \in \operatorname{im} j^{*}$, and $S q^{q+n} \bar{x}=0$ by the unstable condition). Now there is a commutative diagram

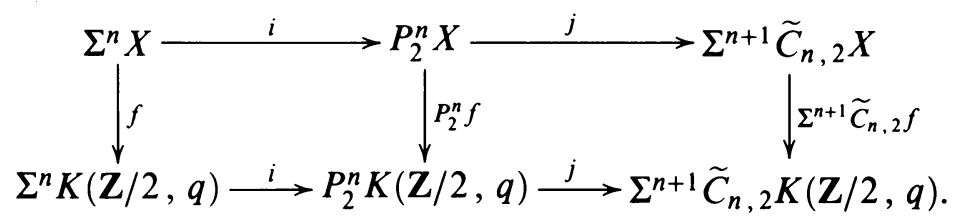

By construction, $f^{*}\left(l_{q}\right)=x,\left(P_{2}^{n} f\right)^{*}\left(\bar{l}_{q}\right)=\bar{x}$, and $\left(\widetilde{C}_{n, 2} f\right)^{*}\left(\widetilde{Q}_{n-1} l_{q}\right)=\widetilde{Q}_{n-1} x$, and the result follows. Q.E.D.

The following corollary is the stable version of Theorem 6.10. Recall from (5.5) that there is a natural map $\Sigma^{-n} \Sigma^{\infty} P_{2}^{n} X \rightarrow \mathbf{P}_{2} X$ for every $x \in \mathrm{Ob} \mathscr{H}_{2}^{\infty}$. 
The geometric filtration (5.6) yields an algebraic filtration of $\widetilde{H}^{*}\left(\mathbf{P}_{2} X ; \mathbf{F}_{2}\right)$, and we define

$$
F^{k+1}=\operatorname{ker}\left\{\widetilde{H}^{*}\left(\mathbf{P}_{2} X ; \mathbf{F}_{2}\right) \rightarrow \widetilde{H}^{*}\left(\Sigma^{-k} \boldsymbol{\Sigma}^{\infty} P_{2}^{k} X ; \mathbf{F}_{\mathbf{2}}\right\} .\right.
$$

Corollary 6.11. Suppose $X \in \mathrm{Ob} \mathscr{H}_{2}^{\infty}$, with basic cofibration sequence

$$
\boldsymbol{\Sigma}^{\infty} \tilde{C}_{\infty, 2} X \stackrel{h}{\longrightarrow} \boldsymbol{\Sigma}^{\infty} X \stackrel{i}{\rightarrow} \mathbf{P}_{\mathbf{2}} X \stackrel{j}{\longrightarrow} \boldsymbol{\Sigma} \boldsymbol{\Sigma}^{\infty} \tilde{C}_{\infty, 2} X .
$$

If $\bar{x} \in \widetilde{H}^{q}\left(\mathbf{P}_{\mathbf{2}} X ; \mathbf{F}_{\mathbf{2}}\right)$, then

$$
S q^{q+n} \bar{x}=j^{*}\left(\sigma^{\infty}\left(\widetilde{Q}_{n-1} x\right)\right) \bmod F^{n+1},
$$

where $i^{*} \bar{x}=\sigma^{\infty} x$ defines $x \in \widetilde{H}^{q}\left(X ; \mathbf{F}_{2}\right)$. In particular, if $S q^{q+n} \bar{x}=0$ $\bmod F^{n+1}$, then there is an element $y \in \widetilde{H}^{2 q+n-1}\left(X ; \mathbf{F}_{\mathbf{2}}\right)$ satisfying $\langle x, v\rangle=$ $\left\langle y, Q_{n-1} v\right\rangle$ for all $v \in \widetilde{H}_{q}\left(X ; \mathbf{F}_{2}\right)$.

The Nishida relations [Ni, CLM] are formulas which relate the action of Dyer-Lashof operations to the (adjoint) action of the Steenrod algebra on $\widetilde{H}_{*}\left(\widetilde{C}_{n, 2} X ; \mathbf{F}_{2}\right)$. The analogous formulas in cohomology are given in the next proposition.

Proposition 6.12. The following formula holds for every $x \in \widetilde{H}^{q}\left(X ; \mathbf{F}_{2}\right)$ and every $r>0$ :

$$
S q^{k} \widetilde{Q}_{r} x=\sum_{i=0}^{[k / 2]}\left(\begin{array}{c}
q+r-i \\
k-2 i
\end{array}\right) \widetilde{Q}_{k+r-2 i} S q^{i} x .
$$

When $r=0$, the formula is true modulo elements dual to external Pontryagin product elements.

Proof. It suffices to prove the formulas when $X=K(\mathbf{Z} / 2, q)$ and $x=l_{q}$, where they are dual to the usual Nishida formulas. Q.E.D.

\section{EXAMPLES AND FURTHER REMARKS}

Example 7.1. $[Z / 2, X]_{\mathscr{E}_{2}^{1}}$.

A pleasant example using our basic definitions arises by considering $Z / 2$ as a two-point $\mathrm{H}$-space. By inspection of the definitions one sees that, for any H-space $X$, the space of $H_{2}^{1}$-functions $H_{2}^{1}(\mathbf{Z} / 2, X)$ can be identified with the homotopy fiber of the $\mathrm{H}$-space squaring map 2: $X \rightarrow X$. Thus there is an exact sequence

$$
\pi_{1}(X) \stackrel{2}{\rightarrow} \pi_{1}(X) \rightarrow[\mathrm{Z} / 2, X]_{\mathscr{H}_{2}^{1}} \rightarrow \pi_{0}(X) \stackrel{2}{\longrightarrow} \pi_{0}(X) .
$$

Also, if $X=\Omega Y$, then $H_{2}^{1}(\mathbf{Z} / 2, \Omega Y)=Y^{\mathbf{R} P^{2}}$, so that $[\mathrm{Z} / 2, \Omega Y]_{\mathscr{P _ { 2 }}}=$ $\left[\mathbf{R} P^{2}, Y\right]$, as predicted by Theorem 4.9 .

Remark 7.2. The Miller spectral sequence and $H_{\infty}^{\infty}$-maps.

If $X$ is an infinite loop space with structure map $\theta_{\infty}^{X}: C_{\infty, \infty} X \rightarrow X$ and associated spectrum $\mathbf{B}^{\infty} X$, and $E^{*}$ a generalized cohomology theory, there is a spectral sequence for computing $E^{*}\left(\mathbf{B}^{\infty} X\right)$ based on knowledge of $E^{*}(X)$ [Mi, Ma]. In particular, the edge homomorphism corresponds to $\epsilon^{*}: E^{*}\left(\mathbf{B}^{\infty} X\right) \rightarrow$ $E^{*}(X)$. Let $E_{q}$ denote the $q$ th space of the spectrum representing $E^{*}$. 
Proposition 7.3. Suppose $x \in E^{q}(X)=E_{1}^{0, q}$ is represented by $f: X \rightarrow E_{q}$. Then $x$ lives to $E_{2}^{0, q}$ if and only if $f$ admits an $H_{\infty}^{\infty}$-structure.

Proof. $f$ admits an $H_{\infty}^{\infty}$-structure if and only if

$$
x \in \operatorname{ker}\left\{h^{*}: E^{q}(X) \rightarrow E^{q}\left(\tilde{C}_{\infty, \infty} X\right)\right\}
$$

if and only if

$$
x \in \operatorname{ker}\left\{\left(\theta_{\infty}^{X}\right)^{*}-\varepsilon_{\infty}^{*}: E^{q}(X) \rightarrow E^{q}\left(C_{\infty, \infty} X\right)\right\} .
$$

But by construction of the spectral sequence, $d_{1}=\left(\theta_{\infty}^{X}\right)^{*}-\varepsilon_{\infty}^{*}$. Q.E.D.

Remark 7.4. The Kahn-Priddy theorem and $H_{p}^{\infty}$-maps.

The generalized Kahn-Priddy theorem proved by the first author in [Ku2] admits the following reinterpretation in our setting.

Theorem 7.5. Suppose $f: X \rightarrow Y$ is a map between connected infinite loop spaces, localized at a prime $p$. If $f$ admits an $H_{p}^{\infty}$-structure, then it admits an $H_{\infty}^{\infty}$-structure.

Proof. If $Y=\Omega^{\infty} \mathbf{E}$, then the adjoint of $f$ is a map $\hat{f}: \mathbf{\Sigma}^{\infty} X \rightarrow \mathbf{E} . f$ admits an $H_{p}^{\infty}$-structure if and only if $\hat{f}$ extends to $\mathbf{P}_{\mathbf{p}} X$, and $f$ admits an $H_{\infty}^{\infty}$ structure if and only if $\hat{f}$ extends to $\mathbf{P}_{\infty} X$. The Kahn-Priddy theorem of [Ku2] (reinterpreted using Corollary 5.2) essentially shows that, localized at $p$, there is a map $s: \Sigma^{\infty} \widetilde{C}_{\infty, \infty} X \rightarrow \Sigma^{\infty} \widetilde{C}_{\infty, p} X$ making the diagram

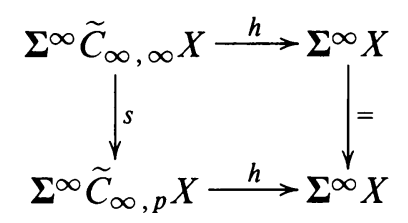

homotopy commute. Thus there is a map $t: \mathbf{P}_{\infty} X \rightarrow \mathbf{P}_{\mathbf{p}} X$ making the diagram

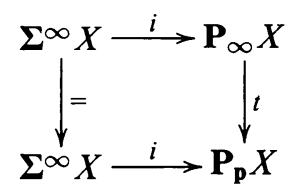

commute. Q.E.D.

The ordinary Kahn-Priddy theorem, in its "once delooped" version, is the special case $X=S^{1}$.

Example 7.6. $\mathbf{P}_{2} S^{0}$.

Viewing $S^{0}$ as $\mathbf{Z} / 2=K(\mathbf{Z} / 2,0)$, and noting that $\widetilde{C}_{\infty, 2} S^{0}=\mathbf{R} P_{+}^{\infty}$, our basic sequence takes the form

$$
\boldsymbol{\Sigma}^{\infty} \mathbf{R} P_{+}^{\infty} \stackrel{h}{\longrightarrow} \boldsymbol{\Sigma}^{\infty} S^{0} \stackrel{i}{\longrightarrow} \mathbf{P}_{2} S^{0} \stackrel{j}{\longrightarrow} \boldsymbol{\Sigma}^{\infty} \boldsymbol{\Sigma} \mathbf{R} P_{+}^{\infty} .
$$

There is a bottom class $\bar{x} \in \widetilde{H}^{0}\left(\mathbf{P}_{2} S^{0} ; \mathbf{F}_{\mathbf{2}}\right)$ such that $x=i^{*}(\bar{x})$ is the nonzero class in $\widetilde{H}^{0}\left(S^{0} ; \mathbf{F}_{2}\right)$. It is easy to see that $\widetilde{Q}_{r} x \in H^{r}\left(\mathbf{R} P^{\infty}, \mathbf{F}_{\mathbf{2}}\right)$ is the nonzero 
class for all $r \geq 0$, and thus Corollary 6.11 implies that $S q^{n} \bar{x} \neq 0$ for all $n \geq 0$.

If we consider maps from $\mathbf{Z} / 2$ into $\Omega^{k} Q S^{0}$, then we obtain the following interesting interpretation. The transfer $\boldsymbol{\Sigma}^{\infty} \mathbf{R} P_{+}^{\infty} \stackrel{h}{\longrightarrow} \boldsymbol{\Sigma}^{\infty} S^{0}$ induces a monomorphism in negative degrees in stable cohomotopy, which means $i^{*}\left[\mathbf{P}_{2} S^{0}, \mathbf{S}^{-\mathbf{k}}\right]=$ 0 . Thus by Theorem 4.9 , there are no essential $H_{2}^{\infty}$-maps from $\mathbf{Z} / 2$ into $\Omega^{\infty} \mathbf{S}^{-\mathbf{k}}=\Omega^{k} Q S^{0}$. Given a nonzero element of $x \in \pi_{k}^{S}$, consider it as a map $f: \mathbf{Z} / 2 \rightarrow \Omega^{k} Q S^{0}$. Let $n$ be the largest integer such that there exists an $H_{2}^{n}$-structure $F$ for $f$. By Corollary 6.3, the obstruction to extending $F$ to an $H_{2}^{n+1}$-structure is a nonzero element $M_{F}(x) \in\left[\boldsymbol{\Sigma}^{\infty}(\mathbf{Z} / 2 \wedge \mathbf{Z} / 2), \mathbf{S}^{-\mathbf{k}-\mathbf{n}}\right]=\pi_{n+k}^{S}$. We have the following observation.

Proposition 7.7. The elements $M_{F}(x)$, as $F$ runs through all of the $H_{2}^{n}$-structures for $f$, are the Mahowald root invariant elements associated to the homotopy class $x$.

Proof. It is straightforward from the definitions after taking $(-1)$ duals. Q.E.D.

We end this section with two examples of how one can use Theorem 6.10. These results are easy and known, but we give them to illustrate the methods developed in $\S 6$. For a deeper application of these methods, the reader can consult [S12].

Example 7.8. The three sphere $S^{3}$ is not homotopy commutative, i.e. is not an object in $\mathscr{H}_{2}^{2}$.

Suppose $S^{3}$ were an object of $\mathscr{H}_{2}^{2}$. Then one would have the sequence

$$
\Sigma^{2} S^{3} \stackrel{i}{\longrightarrow} P_{2}^{2} S^{3} \stackrel{j}{\longrightarrow} \Sigma^{3} \widetilde{C}_{2,2} S^{3} \stackrel{h}{\longrightarrow} \Sigma^{3} S^{3} .
$$

By connectivity arguments, it is easy to see that $\widetilde{H}^{6}\left(P_{2}^{2} S^{3} ; \mathbf{F}_{\mathbf{2}}\right)=\widetilde{H}^{8}\left(P_{2}^{2} S^{3} ; \mathbf{F}_{2}\right)$ $=0$. For dimensional reasons, $j^{*}\left(\sigma^{2}\left(\widetilde{Q}_{1} x_{3}\right)\right) \neq 0$ (where $x_{3} \in \widetilde{H}^{3}\left(S^{3} ; \mathbf{F}_{2}\right)$ is the nonzero class), so that $S q^{5} \bar{x}_{3} \neq 0$. But

$$
S q^{5} \bar{x}_{3}=S q^{2} S q^{3} \bar{x}_{3}+S q^{4} S q^{1} \bar{x}_{3}=0
$$

since $S q^{3} \bar{x}_{3} \in \widetilde{H}^{8}\left(P_{2}^{2} S^{3} ; \mathbf{F}_{2}\right)$ and $S q^{1} \bar{x}_{3} \in \widetilde{H}^{6}\left(P_{2}^{2} S^{3} ; \mathbf{F}_{2}\right)$. This contradiction shows that $S^{3}$ is not in $\mathscr{H}_{2}^{2}$.

Example 7.9. $S^{3}\langle 3\rangle$ is not an $H_{2}^{4}$-space.

The reader is invited to compare this with the same example in [S11], except in that case it is done from the point of view of obstruction theory and secondary operations. Let $S^{3}\langle 3\rangle$ be the 3-connective cover of $S^{3}$. This is an $\mathrm{H}$-space obtained by killing the generator of $H^{3}\left(S^{3} ; \mathbf{Z}\right)$. The important thing to know is that the $\bmod 2$ cohomology is given (as an algebra) by

$$
H^{*}\left(S^{3}\langle 3\rangle ; \mathbf{F}_{2}\right) \cong \mathbf{F}_{2}\left(x_{4}\right) \otimes \Lambda\left(x_{5}\right)
$$

where $S q^{1} x_{4}=x_{5}$.

Suppose that $S^{3}\langle 3\rangle$ were an $H_{2}^{4}$-space. Then we would have the sequence

$$
\Sigma^{4} S^{3}\langle 3\rangle \stackrel{i}{\longrightarrow} P_{2}^{4} S^{3}\langle 3\rangle \stackrel{j}{\longrightarrow} \Sigma^{5} \widetilde{C}_{4,2} S^{3}\langle 3\rangle \stackrel{h}{\longrightarrow} \Sigma^{5} S^{3}\langle 3\rangle .
$$


Since $P H^{13}\left(S^{3}\langle 3\rangle ; \mathbf{F}_{2}\right)=0$, the element $\sigma^{5}\left(\widetilde{Q}_{3} x_{5}\right)$ is not in the image of $h^{*}$. Thus Theorem 6.10 implies

$$
S q^{9} \bar{x}_{5}=j^{*}\left(\sigma^{5}\left(\widetilde{Q}_{3} x_{5}\right)\right) \neq 0 .
$$

Using the Adem relation

$$
S q^{9}=\left(S q^{8}+S q^{4} S q^{4}\right) S q^{1}+\left(S q^{7}+S q^{4} S q^{2} S q^{1}\right) S q^{2},
$$

we need to check the possible values of $S q^{1} \bar{x}_{5}$ and $S q^{2} \bar{x}_{5}$. Clearly they go to zero in $H^{*}\left(\Sigma^{4} S^{3}\langle 3\rangle ; \mathbf{F}_{2}\right)$ under the map $i^{*}$ for dimensional reasons. Thus they must be in the image of $j^{*}$. Since the degrees of these elements are 10 and 11 , respectively, and $S^{3}\langle 3\rangle$ is 3-connected, connectivity arguments show that there are no nonzero classes in $\widetilde{H}^{*}\left(\Sigma^{5} \widetilde{C}_{4,2} S^{3}\langle 3\rangle ; \mathbf{F}_{2}\right)$ which can map to these elements. Therefore $S q^{1} \bar{x}_{5}$ and $S q^{2} \bar{x}_{5}$ are zero in $\widetilde{H}^{*}\left(P_{2}^{4} S^{3}\langle 3\rangle ; \mathbf{F}_{2}\right)$. This is a contradiction; thus it must be the case that $S^{3}\langle 3\rangle$ is not an $H_{2}^{4}$-space.

\section{APPENDIX A}

In Corollary 3.8, we noted that for an H-space $X$ with multiplication map $\theta: X \times X \rightarrow X$, the map $h$ is the unique map making the diagram

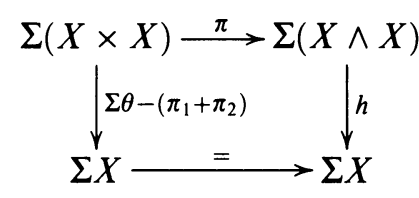

homotopy commute.

In this appendix, we will define and characterize the Hopf construction $H$ : $X * X \rightarrow \Sigma X$ in a similar manner. This characterization will lead to the fact that the maps $h$ and $H$ agree up to homotopy after one suspension. We note that the facts proved here about $H$ are known, but we give complete details for lack of an adequate reference.

Let $s: \Sigma(X \wedge Y) \rightarrow \Sigma(X \times Y)$ be the unique map (up to homotopy) making the diagram

$$
\begin{gathered}
\Sigma(X \times Y) \stackrel{\pi}{\longrightarrow} \Sigma(X \wedge Y) \\
\downarrow-\Sigma i_{y} \pi_{Y}+\Sigma \text { id }-\Sigma i_{X} \pi_{X} \downarrow s \\
\Sigma(X \times Y) \stackrel{=}{\longrightarrow} \Sigma(X \times Y)
\end{gathered}
$$

homotopy commute, where $\pi_{X}, \pi_{Y}: X \times Y \rightarrow X, Y$ are the projections and $i_{X}, i_{Y}: X, Y \rightarrow X \times Y$ are the inclusions.

Definition A.1. Given a map $\theta: X \times Y \rightarrow Z$, let $\bar{H}: \Sigma(X \wedge Y) \rightarrow Z$ be the composite $\Sigma \theta \circ s$, where $s$ is the map from diagram (A.2).

Recall that the (reduced) cone on $X$, denoted $C X$, is the quotient obtained from $X \times I$ by collapsing the subspace $X \times\{1\} \cup * \times I$ to a point. The (reduced) join $X * Y$ is defined as the subspace $\left(X \times C_{+} Y\right) \cup_{X \times Y}\left(C_{-} X \times Y\right) \subset C_{-} X \times C_{+} Y$, where $C_{-} X$ and $C_{+} Y$ are the (reduced) cones on $X$ and $Y$, respectively. Note that the suspension $\Sigma Z$ is homeomorphic to $C_{-} Z \cup_{Z} C_{+} Z$. 
Definition A.2. The Hopf construction on a map $\theta: X \times Y \rightarrow Z$ is the map $H(\theta): X * Y \rightarrow \Sigma Z$ which is defined via the two maps $H_{+}: X \times C_{+} Y \rightarrow C_{+} Z$ and $H_{-}: C_{-} X \times Y \rightarrow C_{-} Z$ given by

$$
\begin{aligned}
& H_{+}(x,(t, y))=(t, \theta(x, y)), \\
& H_{-}((t, x), y)=(t, \theta(x, y)) .
\end{aligned}
$$

If we apply Definition A.2 to the identity $X \times Y \rightarrow X \times Y$, we get a map $H($ id $): X * Y \rightarrow \Sigma(X \times Y)$. By inspection, the composite

$$
X * Y \stackrel{H(\mathrm{id})}{\longrightarrow} \Sigma(X \times Y) \stackrel{\pi}{\longrightarrow} \Sigma(X \wedge Y)
$$

is a quotient map in which a subspace homeomorphic to $C_{-} X \vee C_{+} Y$ has been collapsed to a point. Thus the composite is a homotopy equivalence.

Definition A.3. Given $\theta: X \times Y \rightarrow Z$, let $H: \Sigma(X \wedge Y) \rightarrow \Sigma Z$ be defined as the composite

$$
\Sigma(X \wedge Y) \stackrel{(\pi \circ H(\mathrm{id}))^{-1}}{\longrightarrow} X * Y \stackrel{H(\theta)}{\longrightarrow} \Sigma Z
$$

Theorem A.4. Given $\theta: X \times Y \rightarrow Z$, the maps $H, \bar{H}: \Sigma(X \wedge Y) \rightarrow \Sigma Z$ are homotopic.

Proof. It suffices by naturality to verify this for the universal example $\theta=$ id: $X \times Y \rightarrow X \times Y$. In this case, $\bar{H}=s$, and $H=H($ id $) \circ(\pi \circ H(\text { id }))^{-1}$. By the characterization of $s$ given in diagram (A.2), the statement that $H \simeq \bar{H}$ is easily seen to be equivalent to the commutativity (up to homotopy) of the following diagram:

$$
\begin{array}{ccr}
X * Y & = & X * Y \\
\downarrow H(\mathrm{id}) & & \downarrow H(\mathrm{id}) \\
\Sigma(X \times Y) & \stackrel{-\Sigma i_{y} \pi_{Y}+\Sigma \mathrm{id}-\Sigma i_{X} \pi_{X}}{\longrightarrow} & \Sigma(X \times Y) .
\end{array}
$$

Hence it suffices to show that $\left(-\Sigma i_{Y} \pi_{Y}+\Sigma \mathrm{id}-\Sigma i_{X} \pi_{X}\right) \circ H(\mathrm{id}) \simeq(c+\Sigma \mathrm{id}+c) \circ$ $H($ id), where $c: \Sigma(X \times Y) \rightarrow \Sigma(X \times Y)$ sends all points to the basepoint.

For simplicity of notation, denote the composition $\left(-\Sigma i_{Y} \pi_{Y}+\Sigma \mathrm{id}-\Sigma i_{X} \pi_{X}\right)$ 。 $H($ id) by $\widetilde{H}$. Then

$$
\begin{aligned}
& \widetilde{H}(x,(t, y))= \begin{cases}(1-4 t, \theta(x, y)) \in C_{-} Z & \text { if } t \in\left[0, \frac{1}{4}\right] ; \\
(4 t-1, \theta(x, y)) \in C_{+} Z & \text { if } t \in\left[\frac{1}{4}, \frac{1}{2}\right] ; \\
(3-4 t, x) \in C_{+} Z & \text { if } t \in\left[\frac{1}{2}, \frac{3}{4}\right] ; \\
(4 t-3, x) \in C_{-} Z & \text { if } t \in\left[\frac{3}{4}, 1\right] ;\end{cases} \\
& \widetilde{H}((t, x), y)= \begin{cases}(1-2 t, y) \in C_{-} Z & \text { if } t \in\left[0, \frac{1}{2}\right] ; \\
(2 t-1, y) \in C_{+} Z & \text { if } t \in\left[\frac{1}{2}, 1\right]\end{cases}
\end{aligned}
$$

for $(x,(t, y)) \in X \times C_{+} Y$ and $((t, x), y) \in C_{-} X \times Y$. 
Define a homotopy $G:(X * Y) \wedge I_{+} \rightarrow \Sigma(X \times Y)$ by the formula (A.8)

$$
\begin{aligned}
& G((x,(t, y)), s)= \begin{cases}\widetilde{H}(x(t, y)) & \text { if } t \in\left[0, \frac{1}{2}\right] \\
\widetilde{H}\left(x,\left(t-\frac{1}{2} s, y\right)\right) & \text { if } t \in\left[\frac{1}{2}, 1\right] \text { and } 2 t \geq 1+s ; \\
* & \text { if } t \in\left[\frac{1}{2}, 1\right] \text { and } 2 t \leq 1+s\end{cases} \\
& G(((t, x), y), s)= \begin{cases}\widetilde{H}((t-s, x), y) & \text { if } t \geq s ; \\
* & \text { if } t \leq s .\end{cases}
\end{aligned}
$$

One can readily check that $G$ is continuous and gives the desired homotopy $\widetilde{H} \simeq(c+\Sigma$ id $+c) \circ H($ id $)$. Q.E.D.

The reader may have noticed that the homotopy analogous to $G$ above will fail to be continuous if we try to apply it to show

$$
\left(-\Sigma i_{X} \pi_{X}+\Sigma \mathrm{id}-\Sigma i_{Y} \pi_{Y}\right) \circ H(\mathrm{id}) \simeq(c+\Sigma \mathrm{id}+c) \circ H(\mathrm{id})
$$

(i.e. switching the roles of $X$ and $Y$ ). Of course this can be remedied if we exchange $X$ and $Y$ in the definition of the join at the same time. Thus there are actually two canonical choices (depending on how the join is defined) for the Hopf construction.

Theorem A.4 and Corollary 3.8 together admit the following corollary.

Corollary A.5. For an $H$-space $X$, the maps $\Sigma h$ and $\Sigma H$ are homotopic. Proof. The maps in question are maps $\Sigma^{2}(X \wedge X) \rightarrow \Sigma^{2} X$. Since addition of maps out of a double suspension is commutative, the corollary follows easily from the characterizations of $h$ and $H$ in Corollary 3.8 and Theorem A.4. Q.E.D.

Recently, Arkowitz and Silberbush [AS] have completed a very interesting systematic study of the various possible Hopf constructions. In particular, they have shown that there are six distinct versions for the exceptional Lie group $F_{4}$, resulting from all of the possible orders for adding the maps $\Sigma \theta,-\pi_{1}$, and $-\pi_{2}$.

\section{APPENDIX B}

In this appendix we give a short proof of a generalization (to any cohomology theory with products) of Thomas' theorem (see Theorem 6.4) on the cup product structure of the projective plane of an $\mathrm{H}$-space.

As in Appendix A, suppose one is given a map $\theta: X \times Y \rightarrow Z$. Then Definition A.2 gives a map $H: X * Y \rightarrow \Sigma Z$, and we define $I: \Sigma Z \rightarrow P$ and $J: P \rightarrow \Sigma X \wedge \Sigma Y$ by the pushout

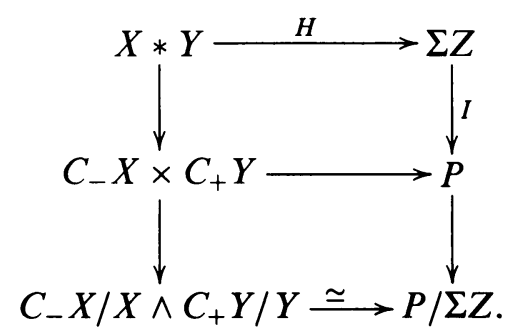


The map $J$ arises as the composite $P \rightarrow P / \Sigma Z \stackrel{\simeq}{\longrightarrow} C_{-} X / X \wedge C_{+} Y / Y \stackrel{\simeq}{\longrightarrow}$ $\Sigma X \wedge \Sigma Y$. Also define $I_{X}: \Sigma X \rightarrow P$ by $\Sigma X \hookrightarrow \Sigma(X \times Y) \stackrel{\Sigma \theta}{\longrightarrow} \Sigma Z \stackrel{I}{\longrightarrow} P$, and similarly, $I_{Y}: \Sigma Y \rightarrow P$.

Proposition B.1. The diagram

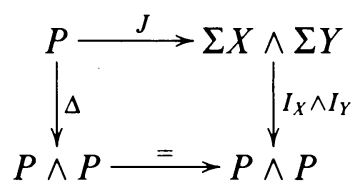

homotopy commutes.

Before proving this, we note the consequences. The following theorem is immediate.

Theorem B.2. Let $E^{*}$ be a generalized (reduced) cohomology theory with products. If $\bar{x}, \bar{y} \in E^{*}(P)$, then

$$
\bar{x} \smile \bar{y}=J^{*}\left(I_{X}^{*}(\bar{x}) \times I_{Y}^{*}(\bar{y})\right) .
$$

Specializing to the H-space situation, we will have $I_{X}=I_{Y}=I$. Thus we obtain the following generalization of Thomas' theorem.

Theorem B.3. Let $\bar{x} \in E^{i+1}\left(P_{2} X\right)$ and $\bar{y} \in E^{j+1}\left(P_{2} X\right)$ define elements $x \in$ $E^{i}(X)$ and $y \in E^{j}(X)$ by $I^{*}(\bar{x})=\sigma x$ and $I^{*}(\bar{y})=\sigma y$. Then

$$
\bar{x} \smile \bar{y}=(-1)^{i} J^{*}\left(\sigma^{2}(x \times y)\right) \text {. }
$$

Note that in combination with Corollary A.5, the case $\bar{x}=\bar{y}$ above specializes to Theorem 6.4 when $E^{*}=\widetilde{H}^{*}\left(; \mathbf{F}_{2}\right)$.

Proof of Proposition B.1. It is convenient to recall the definitions via the following diagram

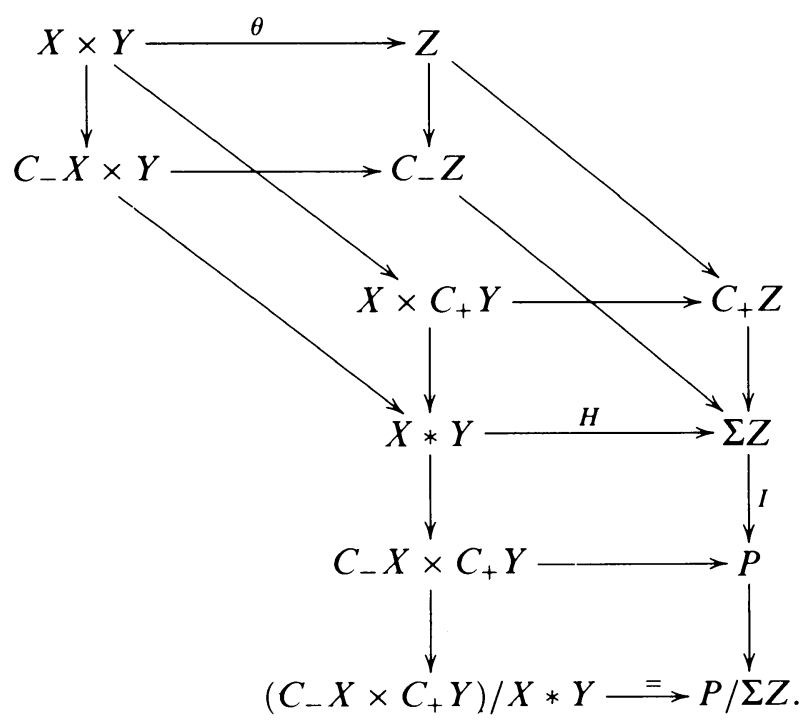

Recall that the composite 
(B.6) $P \rightarrow P / \Sigma Z \stackrel{=}{\longleftarrow}\left(C_{-} X \times C_{+} Y\right) / X * Y \stackrel{\simeq}{\longrightarrow} C_{-} X / X \wedge C_{+} Y / Y \stackrel{\simeq}{\longrightarrow} \Sigma X \wedge \Sigma Y$ yields the map $J$. As result of the definitions we have the following commutative diagram:

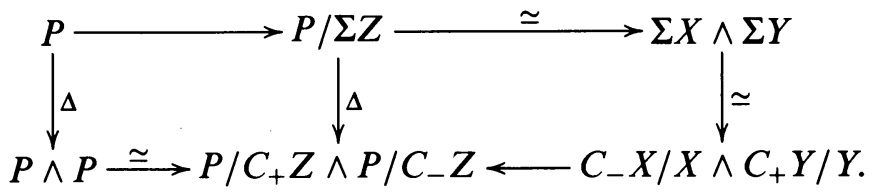

Thus it suffices to verify that the composite

$$
\Sigma X \stackrel{\simeq}{\longrightarrow} C_{-} X / X \rightarrow P / C_{+} Z \stackrel{\simeq}{\longleftarrow} P
$$

is homotopic to $I_{X}$, and similarly for $I_{Y}$.

To show this, we note that the constructions are natural. By applying the definitions to

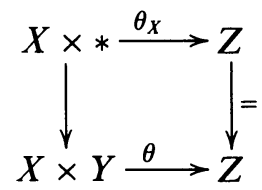

we obtain, up to homotopy

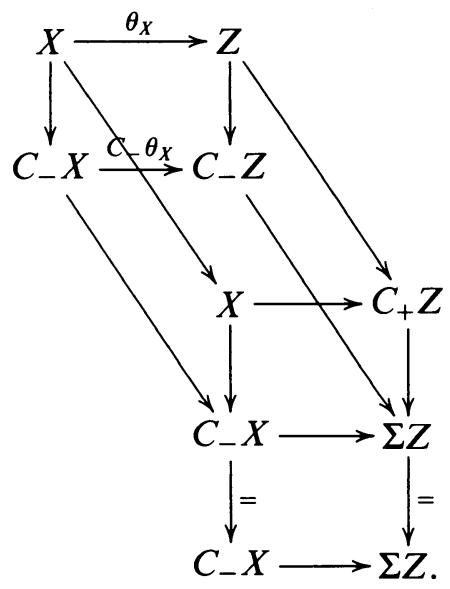

Therefore the composite

$$
\Sigma X \stackrel{\simeq}{\longrightarrow} C_{-} X / X \rightarrow \Sigma Z / C_{+} Z \stackrel{\simeq}{\longleftarrow} Z
$$

is homotopic to $\Sigma \theta_{X}$. Then, by naturality, the composite (B.8) is homotopic to

$$
\Sigma X \stackrel{\Sigma \theta_{X}}{\longrightarrow} \Sigma Z \stackrel{I}{\longrightarrow} P,
$$

which is $I_{X}$ by definition. The proof for $I_{Y}$ is similar. Q.E.D.

\section{REFERENCES}

[Ad] J. F. Adams, On the nonexistence of elements of Hopf invariant one, Ann. of Math. (2) 72 (1960), 20-104. 
[AK] S. Araki and T. Kudo, Topology of $H_{n}$-spaces and $H_{n}$-squaring operations, Mem. Fac. Sci. Kyushu Univ. Ser. A 10 (1956), 85-120.

[AS] M. Arkowitz and P. Silberbush, Some properties of Hopf-type constructions, preprint.

[BV] J. M. Boardman and R. Vogt, Homotopy everything $H$-spaces, Bull. Amer. Math. Soc. 74 (1968), 1117-1122.

[Br] W. Browder, Homology operations and loop spaces, Illinois J. Math. 4 (1960), 347-357.

[BT] W. Browder and E. Thomas, On the projective plane of an $\mathrm{H}$-space, Illinois J. Math. 7 (1963), 492-502.

[CLM] F. R. Cohen, T. J. Lada, and J. P. May, The homology of iterated loop spaces, Lecture Notes in Math., vol. 533, Springer, Berlin and New York, 1976.

[CMT] F. R. Cohen, J. P. May, and L. R. Taylor, Splitting of certain spaces CX, Math. Proc. Cambridge Philos. Soc. 84 (1978), 465-496.

[DL] E. Dyer and R. Lashof, Homology of iterated loop spaces, Amer. J. Math. 84 (1962), 35-88.

[He] Y. Hemmi, The projective plane of an H-pairing, J. Pure Appl. Algebra 75 (1991), 277-296.

[Hu] J. R. Hubbuck, On homotopy commutative H-spaces, Topology 8 (1969), 119-126.

[Ka] R. Kane, Implications in Morava K-theory, Mem. Amer. Math. Soc. 340 (1986).

[Ku1] N. Kuhn, The geometry of the James-Hopf maps, Pacific J. Math. 102 (1982), 397-412.

[Ku2] - Extended powers of spectra and a generalized Kahn-Priddy theorem, Topology 23 (1985), 473-480.

[Li] J. P. Lin, Two torsion and the loop space conjecture, Ann. of Math. (2) 115 (1982), 35-91.

[Mh] M. Mahowald, The metastable homotopy of $S^{n}$, Mem. Amer. Math. Soc. 72 (1967).

[Ma] J. P. May, The geometry of iterated loop spaces, Lecture Notes in Math., vol. 271, Springer, Berlin and New York, 1972.

[Mg1] R. J. Milgram, Iterated loop spaces, Ann. of Math. (2) 84 (1966), 386-403.

[Mg2] - Unstable homotopy from the stable point of view, Lecture Notes in Math., vol. 368, Springer, Berlin and New York, 1974.

[Mi] H. R. Miller, A spectral sequence for the homology of an infinite delooping, Pacific J. Math. 79 (1978), 139-155.

[Ni] G. Nishida, Cohomology operations in iterated loop spaces, Proc. Japan Acad. 44 (1968), 104-109.

[S11] M. Slack, Maps between iterated loop spaces, J. Pure Appl. Algebra 73 (1991), 181-201.

[S12] _ Infinite loop spaces with trivial Dyer-Lashof operations, Math. Proc. Cambridge Philos. Soc. (1993).

[Sf] J. Stasheff, $H$-spaces from the homotopy point of view, Lectures Notes in Math., vol. 161, Springer, Berlin and New York, 1970.

[St] N. Steenrod, A convenient category of topological spaces, Michigan Math. J. 14 (1967), 133-152.

[Th1] E. Thomas, On functional cup operations and the transgression operator, Arch. Math. (Basel) 12 (1961), 435-444.

[Th2] - Steenrod squares and H-spaces. I, II, Ann. of Math. (2) 77 (1963), 306-317; 81 (1965), 473-495.

Department of Mathematics, University of Virginia, Charlottesville, Virginia 22903

E-mail address: njk4xQadams.math.virginia.edu

Department of Mathematics, Stanford University, Stanford, California 94305

E-mail address: slack@math.stanford.edu

Department of Mathematical Sciences, New Mexico State University, las Cruces, New MeXico 88003

E-mail address: frank@nmsu.edu 